Research Article

\title{
Different Angiogenic Potentials of Mesenchymal Stem Cells Derived from Umbilical Artery, Umbilical Vein, and Wharton's Jelly
}

\author{
Lu Xu, ${ }^{1}$ Jianjun Zhou, ${ }^{1}$ Jingyu Liu, ${ }^{1}$ Yong Liu, ${ }^{2}$ Lei Wang, ${ }^{1}$ Ruiwei Jiang, ${ }^{1}$ Zhenyu Diao, ${ }^{1}$ \\ Guijun Yan, ${ }^{1}$ Bruno Pèault, ${ }^{3,4}$ Haixiang Sun, ${ }^{1}$ and Lijun Ding ${ }^{1,5}$ \\ ${ }^{1}$ Center for Reproductive Medicine, Department of Obstetrics and Gynecology, The Affiliated Drum Tower Hospital of Nanjing \\ University Medical School, 321 Zhongshan Rd., Nanjing 210008, China \\ ${ }^{2}$ Central Research Lab, The Affiliated Drum Tower Hospital of Nanjing University Medical School, 321 Zhongshan Rd., \\ Nanjing 210008, China \\ ${ }^{3}$ MRC Center for Regenerative Medicine, University of Edinburgh, Edinburgh EH16 4UU, UK \\ ${ }^{4}$ Orthopedic Hospital Research Center and Broad Stem Cell Center, David Geffen School of Medicine, University of California, \\ Los Angeles, CA, USA \\ ${ }^{5}$ Clinical Center for Stem Cells, The Affiliated Drum Tower Hospital of Nanjing University Medical School, 321 Zhongshan Rd., \\ Nanjing 210008, China
}

Correspondence should be addressed to Haixiang Sun; stevensunz@163.com and Lijun Ding; xmljding@163.com

Received 19 September 2016; Revised 14 February 2017; Accepted 21 February 2017; Published 10 August 2017

Academic Editor: Benedetta Bussolati

Copyright (c) $2017 \mathrm{Lu} \mathrm{Xu}$ et al. This is an open access article distributed under the Creative Commons Attribution License, which permits unrestricted use, distribution, and reproduction in any medium, provided the original work is properly cited.

\begin{abstract}
Human mesenchymal stem cells derived from the umbilical cord (UC) are a favorable source for allogeneic cell therapy. Here, we successfully isolated the stem cells derived from three different compartments of the human UC, including perivascular stem cells derived from umbilical arteries (UCA-PSCs), perivascular stem cells derived from umbilical vein (UCV-PSCs), and mesenchymal stem cells derived from Wharton's jelly (WJ-MSCs). These cells had the similar phenotype and differentiation potential toward adipocytes, osteoblasts, and neuron-like cells. However, UCA-PSCs and UCV-PSCs had more CD146 ${ }^{+}$cells than WJ-MSCs $(P<0.05)$. Tube formation assay in vitro showed the largest number of tube-like structures and branch points in UCA-PSCs among the three stem cells. Additionally, the total tube length in UCA-PSCs and UCV-PSCs was significantly longer than in WJ-MSCs $(P<0.01)$. Microarray, qRT-PCR, and Western blot analysis showed that UCA-PSCs had the highest expression of the Notch ligand Jagged1 (JAG1), which is crucial for blood vessel maturation. Knockdown of Jagged1 significantly impaired the angiogenesis in UCA-PSCs. In summary, UCA-PSCs are promising cell populations for clinical use in ischemic diseases.
\end{abstract}

\section{Introduction}

Over the last few decades, mesenchymal stem cells (MSCs) have been widely explored for their potential as a treatment strategy for disorders caused by insufficient angiogenesis, including atherosclerosis, stroke, myocardial infarction, and chronic wounds [1]. These cells have several characteristic features. First, they can adhere to tissue culture flasks and are positive for specific markers like CD73, CD90, and CD105 and negative for hematopoietic markers such as CD34, CD45, and HLA-DR. Second, they can differentiate into adipocytes, osteoblasts, and chondrocytes in vitro [2]. MSCs can be isolated from many human tissues such as bone marrow, adipose tissue, peripheral blood, dental pulp, placenta, amniotic fluid, umbilical cord (UC), pancreas, and spleen [3-5]. In recent years, UC has been acknowledged to be a better source of MSCs. Besides the noninvasive collection procedure, no ethical issues, and faster self-renewal, UC-derived MSCs have been shown to be multipotent and immunomodulatory [6, 7]. Currently, UC-derived MSCs are isolated primarily from Wharton's jelly (WJ-MSCs), which is the mucoid connective tissue in the UC [8]. 
Actually, there are three large vessels surrounded by the WJ, which is enveloped in the amniotic epithelium, including two umbilical arteries (UCAs) and one umbilical vein (UCV). Previous reports have found that human UC perivascular cells, including UCA perivascular stem cells (UCA-PSCs) and UCV perivascular stem cells (UCV-PSCs), are distinctly different from WJ-MSCs [9]. In particular, $\mathrm{CD}_{146^{+} \mathrm{UC}}$ perivascular cells have been found to express typical MSCs markers and could accelerate wound healing by enhancing angiogenesis $[10,11]$.

MSCs mainly originate from two types of perivascular cells, pericytes $\left(\mathrm{CD} 45^{-} \mathrm{CD} 31^{-} \mathrm{CD} 146^{+} \mathrm{CD} 34^{-}\right)$and adventitial cells $\left(\mathrm{CD} 45^{-} \mathrm{CD} 31^{-} \mathrm{CD} 146^{-} \mathrm{CD} 34^{+}\right)$, which contain the in situ counterpart of MSCs in human organs and yield a progeny of multilineage mesodermal progenitor cells $[12,13]$. Recently, osteogenic and adipogenic progenitors have also been shown to originate from perivascular niches in vivo and purified pericytes [14-16]. In addition, transplantation of purified pericytes can support vasculature and repair damaged tissue $[17,18]$. These results indicate the therapeutic capacity of perivascular stem cells in postinjury angiogenesis/vasculogenesis.

Although many previous studies have identified cell populations arising from specific cord regions, it remains to be unknown if UCA-PSCs, UCV-PSCs, and WJ-MSCs from the same UC differ in terms of proliferation ability, differentiation ability, and especially angiogenic capacity [19-21]. Therefore, we described the basic characterization of UCAPSCs, UCV-PSCs, and WJ-MSCs derived from the same $\mathrm{UC}$ and compared their angiogenic potential in vitro which may provide a new alternative source for cell-based therapeutic applications in ischemia.

\section{Materials and Methods}

2.1. Preparation of Human UC Sample. Human UC tissue samples $(n=10)$ were collected from the Affiliated Drum Tower Hospital of Nanjing University Medical School and processed within $12 \mathrm{~h}$ of natural delivery. The physician obtained verbal informed consent from the healthy mother without any pregnancy complication for the use of the umbilical cord in the present research. The experimental procedure was approved by the Clinical Research Ethics Committee at the Affiliated Drum Tower Hospital of Nanjing University Medical School. The UCs were then immersed in sterile phosphate-buffered saline (PBS, Gibco, Grand Island, NY, USA) supplemented with $5 \%$ penicillin/streptomycin (Gibco) for further tissue analysis or cell isolation.

2.2. Immunofluorescence Assay. UCA, UCV, and WJ were immersed in optimum cutting temperature (OCT) compound (Leica, Wetzlar, Germany) and frozen at $-70^{\circ} \mathrm{C}$ until sectioning. The tissues were serially sectioned to $6 \mu \mathrm{m}$ thickness using a cryostat (Leica). Expression of PDGFR $\beta$ (ab32570, Abcam, Cambridge, UK), NG2 (ab139406, Abcam), $\alpha$-SMA (ab5694, Abcam), and CD146 (ab75769, Abcam) was detected by immunofluorescence staining. After incubated with primary antibody at $4^{\circ} \mathrm{C}$ overnight, the frozen sections were then incubated with Alexa Fluor 488-conjugated goat anti-rabbit IgG $(1: 200$, Invitrogen, Grand Island, NY, USA) or Alexa Fluor 555-conjugated goat anti-rabbit IgG $(1: 200$, Invitrogen). The nuclei were stained with 4',6-diamidino-2-phenylindole (DAPI), which was contained in the Vectashield mounting medium for fluorescence (Vector Laboratories Inc.). The images were visualized using fluorescence confocal microscopy (Leica) under a magnification of 600x. The integrated optical density (IOD) values of positive staining in five randomly selected fields of view were tested by Image pro-plus 6.0 software (Media Cybernetic, Rockville, USA).

2.3. Isolation and Culture of UCA-PSCs, UCV-PSCs, and WJ-MSCs. Adherent cells were isolated and cultured using the explant method. Briefly, two UCAs and one UCV were longitudinally extracted from human UC. The UCA, UCV, and WJ were then manually minced into $1-2 \mathrm{~mm}^{3}$ fragments. The vessels were cut in the direction perpendicular to the long axis with a sterile scissor. These fragments were aligned and seeded regularly on the tissue culture-treated dishes. As to the fragments minced from vessels, only the outlayer but not the cross section could touch the dish. Then, the culture medium containing low-glucose DMEM (LGDMEM; Gibco) supplemented with $10 \%$ fetal bovine serum (FBS; Gibco), 1\% penicillin/streptomycin (Gibco) and $10 \mathrm{ng} / \mathrm{ml}$ basic fibroblast growth factor (FGF2, Gibco) poured slowly and gently, cultured at $37^{\circ} \mathrm{C}$ and $5 \% \mathrm{CO}_{2}$. The culture medium was replaced every 3-5 d for 2 weeks until fibroblast-like adherent cells reach $80-90 \%$ confluence. Then, adherent cells and tissue fragments were rinsed once with PBS and detached using a $0.05 \%$ trypsin/EDTA solution (TE; Gibco). The three types of stem cells were subpassaged at every $4-5 \mathrm{~d}$ with the ratio $1: 4$.

2.4. Proliferation Assay. Cell-counting kit-8 (CCK-8) (Dojindo, Kumarmoto, Japan) was used to measure the cell proliferation. Cells were seeded at $2 \times 10^{3}$ cells per well into 96-cell plates, eight parallel wells for each group, which were conventionally cultured in complete medium (100 $\mu \mathrm{l} /$ well) for every day in one week, respectively. The CCK-8 reagent $(10 \mu \mathrm{l})$ was added into each well. After incubation for $2 \mathrm{~h}$, the optical density value (OD value) was measured at $450 \mathrm{~nm}$ position on a microplate reader (Thermo, Massachusetts, USA). Each well of cells was counted once. The growth curve was draw based on the mean value of the eight counts in each group. The culture medium was taken as blank control.

2.5. Flow Cytometry Analysis. The specific cell surface antigens of cultured cells (passage 3) were analyzed by flow cytometry using a FACScan flow cytometer (Becton Dickinson, USA). Single-cell suspensions were harvested from MSC cultures with $0.05 \%$ trypsin/EDTA (Gibco) and resuspended in PBS. The cells were filtered through a $70 \mu \mathrm{m}$ filter and incubated for $1 \mathrm{~h}$ with fluorescein isothiocyanate- (FITC-) or phycoreythrin- (PE-) conjugated antibodies against human CD13 (eBioscience, Colorado, USA), CD29 (eBioscience), CD34 (BD Pharmingen, San Diego, CA, USA), CD45 (eBioscience), CD73 (BD Pharmingen), 
CD90 (eBioscience), CD105 (eBioscience), CD146 (BD Pharmingen), and HLA-DR (eBioscience).

2.6. Multilineage Differentiation Assay. The cells at passage 3 were assessed for multipotency by adipogenic, osteogenic, and neural-like differentiation assays. Cells were seeded at a density of $5 \times 10^{3} / \mathrm{cm}^{2}$ in 24-well plates and grown in monolayer in DMEM low glucose and FBS (10\%) until reaching $\sim 90 \%$ confluency, and then the cells were given the appropriate differentiation medium.

2.6.1. Adipogenesis. Cells were cultured in adipogenic induction medium (Gibco). On day 14, cultures were stained with oil red O staining (Sigma) as an indicator of intracellular lipid accumulation.

2.6.2. Osteogenesis. Cells were grown in osteogenic induction medium (Gibco) for 21 days. Calcium deposition was shown by alizarin red staining (Sigma, Steinheim, Germany).

2.6.3. Neurogenesis. Cells were treated with the preinduction medium containing $10^{-7} \mathrm{~mol} / \mathrm{L}$ all-trans-retinoic acid (ATRA, Sigma) and $10 \mathrm{ng} / \mathrm{ml}$ FGF2 (Gibco) for $18 \mathrm{~h}$, and then with modified neuronal medium (MNM) for $36 \mathrm{~h}$. The expression of neurofilament medium polypeptide (1:100, sc-16143, Santa Cruz Biotechnology, Santa Cruz, CA, USA) and neuron-specific anolase (1:100, sc-292097, Santa Cruz Biotechnology) in induced MSCs was detected by immunofluorescence staining.

2.7. Tube Formation Assay. Liquid Matrigel (BD Biosciences, USA, BD Matrigel Matrix Cat. No. 356234) was added into 96-well tissue culture plates and polymerized for $30 \mathrm{~min}$ at $37^{\circ} \mathrm{C}$. Cells $\left(1 \times 10^{4} /\right.$ well $)$ were trypsinized, resuspended in serum-free DMEM, and plated onto the top of the Matrigel. HUVECs $\left(1.5 \times 10^{4} /\right.$ well $)$ seeded on the Matrigel bed and cultured in serum-free DMEM containing VEGF $(0.3 \mathrm{nmol} / \mathrm{L})$ were served as the positive control. Following incubation at $37^{\circ} \mathrm{C}$ for $3-24 \mathrm{~h}$, each well was digitally photographed under a microscope (Leica) with phase contrast (magnification: 100x). The observed tubes and branching points were counted. Meanwhile, total tubular length was quantified by ImageJ software (National Institutes of Health, MA, USA) and calculated as the average of the total tubule length from three wells, three to five random fields per well.

2.8. Quantitative Real-Time PCR ( $q R T-P C R)$. Total RNAs were prepared from tissues or cells using TRIzol reagent (Invitrogen, Grand Island, NY, USA) according to the manufacturer's instructions. While the quality of the RNA was evaluated using spectrophotometry and denaturing agarose gel electrophoresis, a $1 \mu \mathrm{g}$ aliquot of purified total RNA was reverse transcribed in a total volume of $20 \mu \mathrm{l}$ using a PrimeScript RT reagent kit (Bio-Rad Laboratories, Hercules, CA, USA). The specific primers used for qRTPCR analysis were as follows: hJagged1, forward 5'-CCT GAAGGGGTGCGGTATAT-3' ${ }^{\prime}$, reverse 5'-GGAGTTGACA CCATCGATGC- $3^{\prime}$ and h18S rRNA, forward 5'-CGGCTA CCACATCCAAGGAA-3', reverse 5'-CTGGAATTACCGC GGCT-3'. Each real-time PCR reaction had the following components: $1 \mu \mathrm{L}$ of RT product, $10 \mu \mathrm{L}$ of SYBR Green PCR Master Mix (Bio-Rad Laboratories), and $500 \mathrm{nM}$ each of the forward and reverse primers. QRT-PCR was performed on a MyiQ Single Color Real-time PCR Detection System (Bio-Rad Laboratories) by the below procedure $\left(95^{\circ} \mathrm{C}, 3 \mathrm{~min}, 94^{\circ} \mathrm{C} 10 \mathrm{~s}, 60^{\circ} \mathrm{C} 30 \mathrm{~s}, 72^{\circ} \mathrm{C} 30 \mathrm{~s}, 40\right.$ cycles). h18S rRNA was used as an internal control for Jagged1 detection. The samples were processed in duplicate using RNA preparations from 3 independent experiments. The fold change in Jagged1 expression was calculated using the $2^{-\Delta \Delta \mathrm{CT}}$ method.

2.9. Western Blot. The cells at passage 3 were rinsed twice with precooled PBS, then $1 \mathrm{~mL}$ of cell lysis buffer $(50.0 \mathrm{mmol} / \mathrm{L}$ Tris $\mathrm{pH}=7.6,150.0 \mathrm{mmol} / \mathrm{L} \mathrm{NaCl}, 0.1 \% \mathrm{SDS}$, $1.0 \% \mathrm{NP}-40$, protease inhibitor cocktail) was added, and the cells were scraped off. The cells were lysed at $4^{\circ} \mathrm{C}$ for $30 \mathrm{~min}$ under rotation and centrifuged at $15000 \mathrm{rpm}$ for $30 \mathrm{~min}$, and the supernatant was collected. Protein concentrations were determined by the BCA Protein Assay Reagent (Thermo Fisher Scientific, Rockford, IL, USA), after which $25 \mu \mathrm{g}$ of total proteins was loaded to $10 \%$ SDS-PAGE gel electrophoresis and transferred to a PVDF membrane (PVDF, Millipore) using the conventional method. The membrane was immunoblotted with primary antibodies against CD146 (1:500, ab75769, Abcam), Jagged1 (1:500, ab109536, Abcam), DLL4 (1:500, ab7280, Abcam), or GAPDH (1:10000, AP0063, Bioworld technology), followed by incubation with a goat anti-mouse $(1: 10000$, BS12478, Bioworld technology) or goat anti-rabbit $(1: 10000$, A0545, Sigma) secondary antibody. The bands were detected using an enhanced chemiluminescence kit (Amersham Biosciences Corp., Piscataway, NJ, USA), and densitometric analysis of each band was performed with Quantity-one (Bio-Rad) software.

2.10. Gene Microarray. Isolation and quality of total RNA were measured according to the above methods. Microarray analysis was used to screen changes in genome-wide gene expression patterns in UCA-PSCs, UCV-PSCs, and WJ-MSCs separated from the same human UC. The changes in 28264 human gene expression patterns were assessed by Phalanx Biotech gene microarray using the Human HOA7.1 One Array Plus (Phalanx Biotech Group, San Diego, CA).

2.11. Small Interfering RNA Transfection. Small interfering RNA (siRNA) was purchased from Ribo Life Science Co., Ltd. The three stem cells were transfected with Jagged 1 siRNA (si-Jagged1) $(50 \mathrm{nM})$ or negative control siRNA $(\mathrm{si}-\mathrm{NC})(50 \mathrm{nM})$ in mediation of Lipofectamine ${ }^{\mathrm{TM}} 2000$ Transfection Reagent (Invitrogen Inc., Carlsbad, CA, USA). Cells in each group were seeded in a 6-well plate and cultured in an incubator at $37^{\circ} \mathrm{C}$ with $5 \% \mathrm{CO}_{2}$ until $80 \%$ confluence. Cell transfection was performed strictly according to the operation manual of Lipofectamine 2000 Transfection Reagent. The knockdown efficiency was confirmed at $48 \mathrm{~h}$ and $72 \mathrm{~h}$ posttransfection by RT-qPCR and western blot analysis, respectively. Then, in vitro angiogenic 
properties of the three stem cells were determined at $72 \mathrm{~h}$ after transfection.

2.12. Statistical Analysis. Each experiment was repeated at least 3 times. All values were expressed as the means \pm standard error (SE). A two-tailed Student's $t$-test was used to evaluate the differences between two groups. The statistical significance of the difference among multiple comparisons was determined by one-way analysis of variance using Statistics Package for Social Science (SPSS 22.0, SPSS, Chicago, IL, USA). A $P$ value $<0.05$ was considered statistically significant.

\section{Results}

3.1. Expression of PDGF-R $\beta, N G 2, \alpha-S M A$, and CD146 in Human UC. PDGF-R $\beta$ is a platelet-derived growth factor receptor which is involved in pericyte formation and recruitment during blood vessel morphogenesis. NG2 is a proteoglycan associated with pericytes during vascular morphogenesis. $\alpha$-SMA can be reproducibly detected in cells surrounding the venules and arterioles and is responsible for regulating microvessel contractility. CD146 is an endothelial cell antigen expressed at the surface of pericytes [12]. In this study, immunofluorescence staining was used to visualize the expression of PDGF-R $\beta$, NG2, $\alpha$-SMA, and CD146 in UCA, UCV, and WJ samples obtained from the same UC. The results revealed high expression of PDGF-R $\beta$ in the perivascular region while PDGF-R $\beta^{+}$cells were scarcely detected in the WJ (Figures 1(a), 1(b), and $1(\mathrm{c})$ ). $\mathrm{NG}^{+}$cells were primarily distributed in the UCA, followed by the UCV, while there was almost no NG2 ${ }^{+}$cells in WJ (Figures $1(\mathrm{~d}$ ), $1(\mathrm{e})$, and $1(\mathrm{f})) . \alpha$-SMA staining revealed a similar distribution pattern (Figures 1(g), 1(h), and 1(i)). These data demonstrated that pericyte markers (PDGF-R $\beta$, NG2, and $\alpha$-SMA) were detected primarily in the perivascular region. CD146 expression was highly prevalent in the perivascular region, especially in the UCA (Figure 1(j)), followed by UCV (Figure 1(k)), but CD146 expression in the WJ was very low (Figure 1(l)). Quantitative analysis of the immunostaining showed that there were more PDGF-R $\beta^{+}$(Figure $1(\mathrm{~m})$ ), $\mathrm{NG}^{+}$(Figure $1(\mathrm{n})$ ), $\alpha$-SMA ${ }^{+}$(Figure $1(\mathrm{o})$ ), and $\mathrm{CD} 146^{+}$ (Figure 1(p)) cells in the perivascular region than in WJ which suggested that the UCA and UCV walls contained most pericytes of UC.

3.2. Phenotypes of UCA-PSCs, UCV-PSCs, and WJ-MSCs. UCA-PSCs, UCV-PSCs, and WJ-MSCs were isolated from human UC using tissue explants. The obtained UCA, UCV, and $\mathrm{WJ}$ tissue samples were cut into small fragments and plated in dishes (Figures 2(a), 2(b), 2(c), 2(d), 2(e), and 2(f)). On days 7-10 after incubation, fibroblast-like cells migrated out of the tissues (Figures 2(g), 2(h), and 2(i)). The morphology of stem cells derived from the three different tissues was similar (Figures 2(j), 2(k), and 2(l)).

CCK-8 assays showed that UCA-PSCs, UCV-PSCs, and WJ-MSCs had similar proliferation tendency at passage 3 (Figure 3(a)). However, on days 4 and 5, UCA-PSCs had a significantly higher growth rate compared to UCV-PSCs.
Flow cytometric analysis of cells at passage 3 revealed that all these three cell populations were positive for CD13, CD29, CD73, CD90, and CD105 but negative for CD34, CD45, and HLA-DR (Figure 3(b)), which was consistent with the previous reports on MSC surface markers [1]. However, UCA-PSCs and UCV-PSCs had more CD146 ${ }^{+}$cells than WJ-MSCs $(P<0.05$; Figures 3(b), 3(c), 3(d), 3(e), 3(f), 3(g), and $3(\mathrm{~h}))$. CD146 ${ }^{+}$cells in many human tissues have been identified as MSC origin in vivo and have higher multilineage differentiation potential [22].

3.3. Multilineage Differentiation Potential of UCA-PSCs, UCV-PSCs, and WJ-MSCs. To study whether the MSCs derived from perivascular regions and WJ had similar multilineage differentiation capacity, cells from passage 3 were cultured under various conditions for adipogenic, osteogenic, and neural-like differentiation. For adipogenic differentiation, lipid-containing cells were detected earlier in UCA-PSCs and UCV-PSCs than in WJ-MSCs (day 10, day 10, and day 12, resp.; data not shown). At 14 days after induction, the three stem cell populations were all capable of differentiating into adipocytes containing lipid droplets (Figures 4(a), 4(b), and 4(c)). For osteogenesis, bone nodules were first detected in UCA-PSCs and UCV-PSCs but not in WJ-MSCs after the cell populations were cultured under osteogenic conditions on day 10 (data not shown). Three weeks later, alizarin red $\mathrm{S}$ staining revealed a greater extent of mineralization with detectable bone nodules in all three stem cell populations (Figures 4(d), 4(e), and 4(f)). The neural-like differentiation of the stem cells was confirmed by NF-M (Figures 4(g), 4(h), and 4(i)) and NSE (Figures 4(j), 4(k), and 4(l)) using immunofluorescence staining. There were no differences in neural differentiation capacity among the three MSCs. These results suggested that UCA-PSCs, UCV-PSCs, and WJ-MSCs all had multilineage differentiation potential, but UCA-PSCs and UCV-PSCs clearly had a higher ability toward mesoderm lineage differentiation.

3.4. UCA-PSCs Exhibited Better Angiogenesis Capacity In Vitro. To compare angiogenesis capacity between UCAPSCs, UCV-PSCs, and WJ-MSCs, tube formation assays were carried out to investigate the capacity of differentiation into a capillary-like structure. As shown in Figures 5(a), 5(b), and 5(c), UCA-PSCs, UCV-PSCs, and WJ-MSCs were cultured on Matrigel-coated plates for $3 \mathrm{~h}$. Microscopic observation revealed that the number of tubules per random field was apparently higher in UCA-PSCs and UCV-PSCs than in WJ-MSCs. At $6 \mathrm{~h}$, the tubes had partly disintegrated in all three cell populations (Figures 5(d), 5(e), and 5(f)). Meanwhile, tube formation in HUVECs was observed as positive control (Supplemental Figure 1 available online at https://doi.org/10.1155/2017/3175748). Interestingly, tubelike structures remained in UCA-PSCs even after $12 \mathrm{~h}$ on Matrigel but disappeared in UCV-PSCs and WJ-MSCs (Figures 5(g), 5(h), and 5(i)), indicating that UCA-PSCs had advantages over UCV-PSCs and WJ-MSCs in maintaining the stability of the formed tubes. At $24 \mathrm{~h}$, all the tube-like structures degraded in these three stem cell populations 


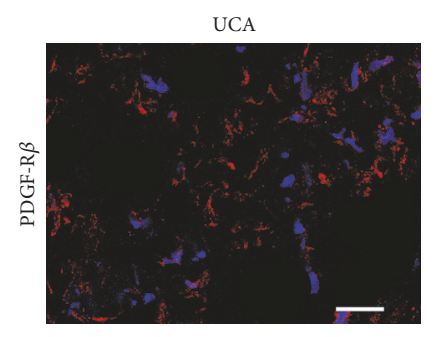

(a)

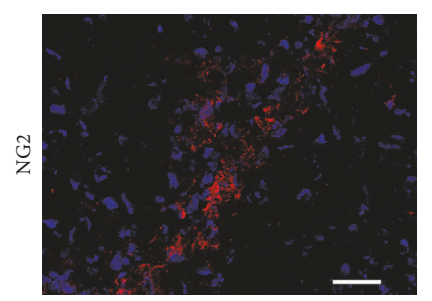

(d)

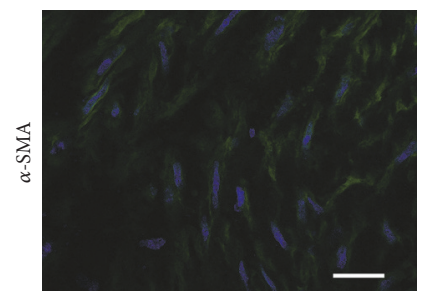

(g)

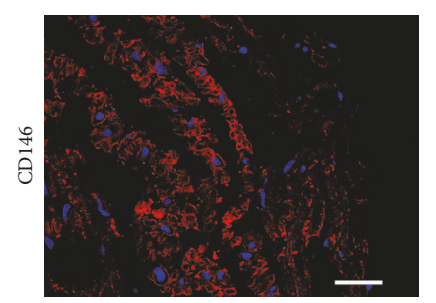

(j)

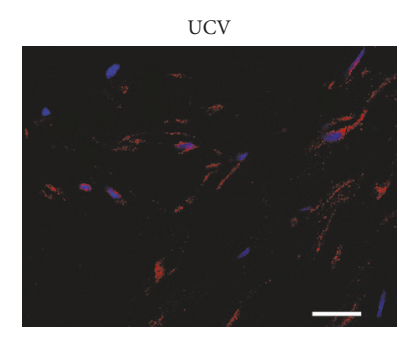

(b)

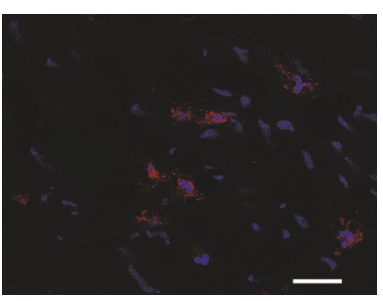

(e)

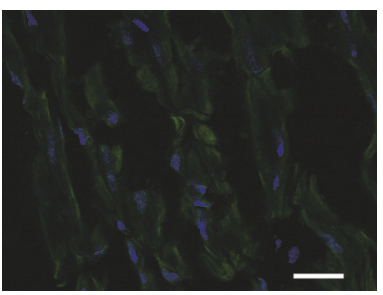

(h)

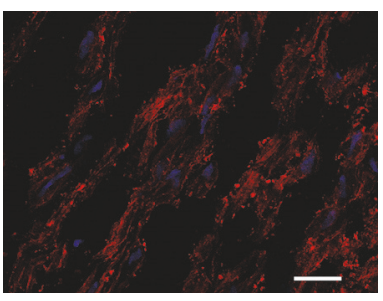

(k)

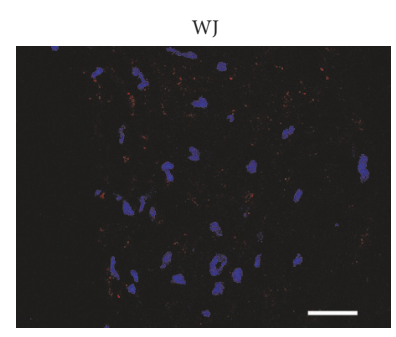

(c)

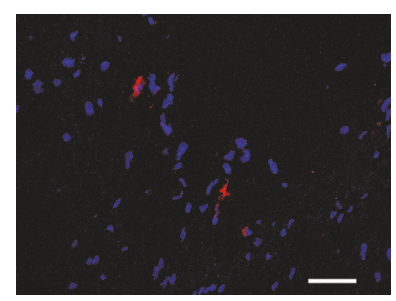

(f)

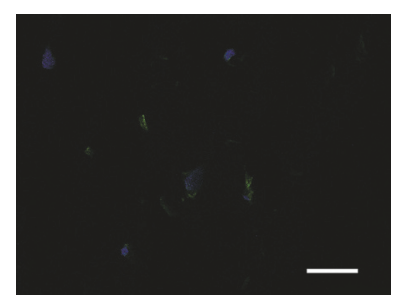

(i)

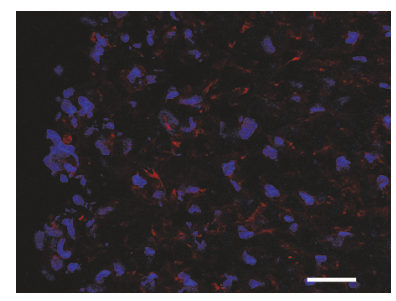

(1)

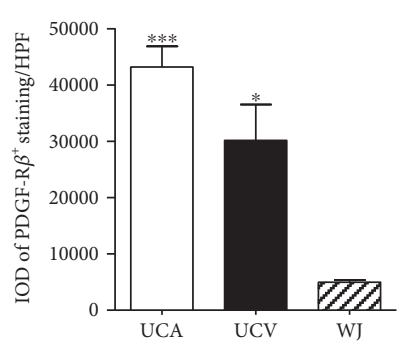

(m)

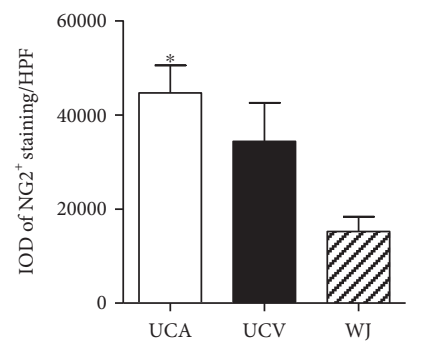

(n)

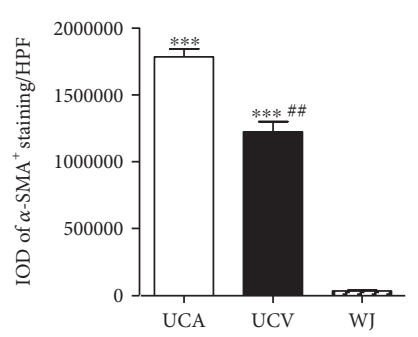

(o)

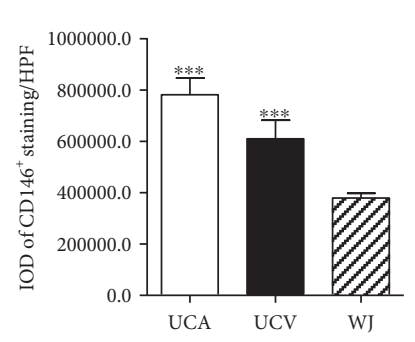

$(\mathrm{p})$

FIGURE 1: Immunolocalization of PDGF-R $\beta$, NG2, $\alpha$-SMA, and CD146 in the human umbilical cord. Fluorescence imaging revealed a high incidence of PDGF-R $\beta$-positive $(\mathrm{a}-\mathrm{c})$, NG2-positive $(\mathrm{d}-\mathrm{f}), \alpha$-SMA-positive (g-i), and CD146-positive (j-l) cells in the perivascular region. Bar: $25 \mu \mathrm{m}$. The integrated optical density (IOD) values of positive staining in five randomly selected high power fields of view were counted. ${ }^{* * *} P<0.001$, versus WJ-MSCs. ${ }^{*} P<0.05$, versus WJ-MSCs. ${ }^{\# \#} P<0.01$, UCV-PSCs versus UCA-PSCs.

(Figures 5(j), 5(k), and 5(l)). Statistical analysis showed that the number of tubes per field significantly increased in UCA-PSCs $(11.08 \pm 1.29)$ and UCV-PSCs $(7.42 \pm 0.57)$ compared to those in WJ-MSCs $(4.33 \pm 0.47 ; P<0.001$; Figure $5(\mathrm{~m}))$. In addition, the number of tube branch points per field was higher in UCA-PSCs $(15.42 \pm 1.14)$ and UCV-PSCs $(11.83 \pm 0.79)$ than in WJ-MSCs $(9.33 \pm 2.87$;
$P<0.001$, UCA-PSCs versus WJ-MSCs; Figure 5(n)). Meanwhile, the total tube length was significantly longer in UCA-PSCs $(199.27 \% \pm 18.90 \% ; P<0.001$; Figure $5(\mathrm{o}))$ and UCV-PSCs $(168.53 \% \pm 9.09 \% ; P<0.01$; Figure $5(\mathrm{o}))$ compared to that in WJ-MSCs. Moreover, the number of tube branch points per field was significantly higher in UCA-PSCs than in UCV-PSCs $(P<0.01)$, while there were 


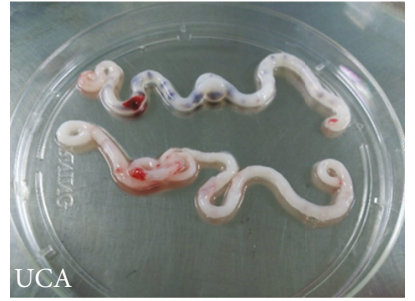

(a)

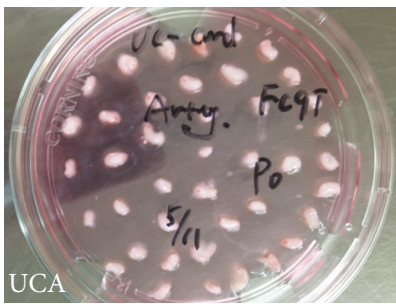

(d)

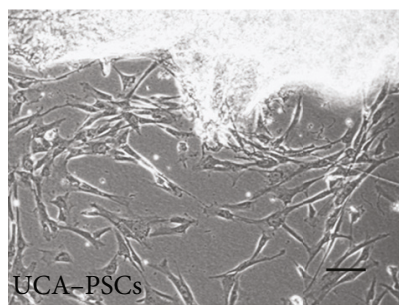

(g)

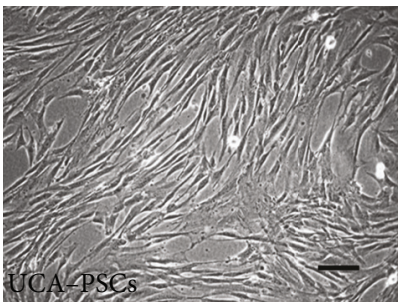

(j)

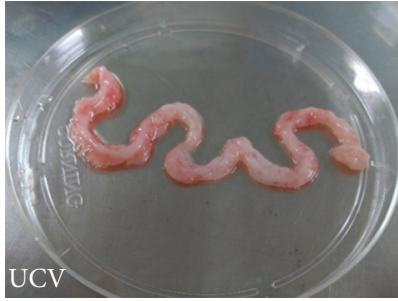

(b)

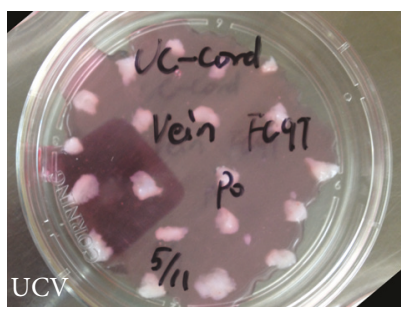

(e)

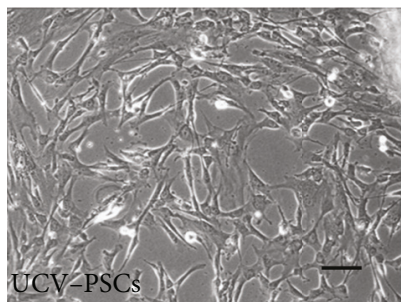

(h)

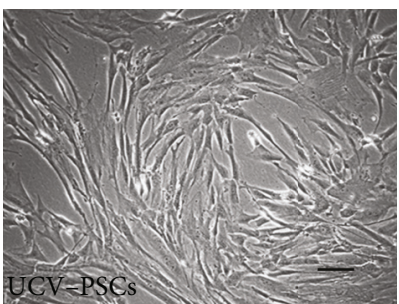

(k)

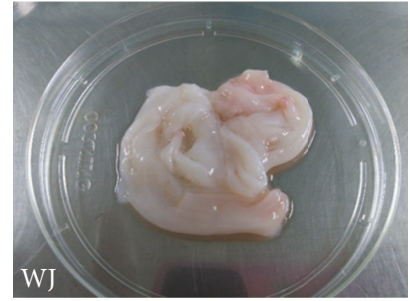

(c)

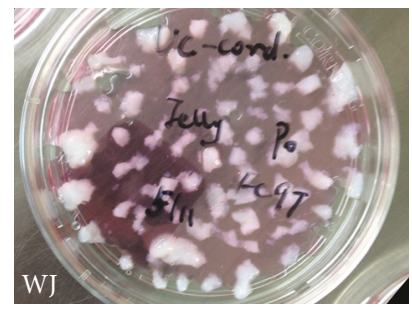

(f)

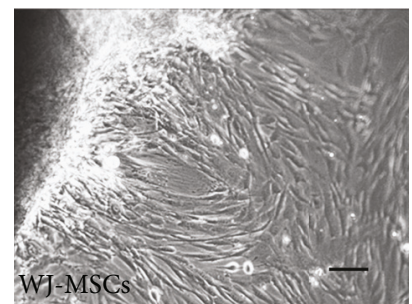

(i)

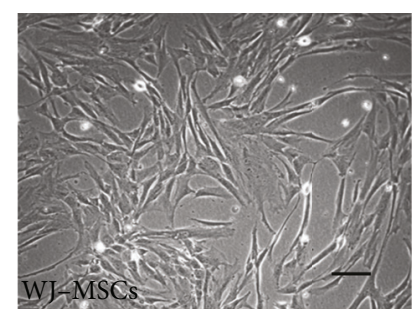

(l)

FIGURE 2: Isolation and characterization of umbilical cord artery perivascular stem cells (UCA-PSCs), umbilical cord vein perivascular stem cells (UCV-PSCs), and Wharton's jelly mesenchymal stem cells (WJ-MSCs). (a-c) Three different compartments in human umbilical cord: umbilical arteries (UCA) (a), umbilical vein (UCV) (b), and Wharton's jelly (WJ) (c). (d-f) Isolation of UCA-PSCs (d), UCV-PSCs (e), and WJ-MSCs in the human umbilical cord (f). (g-i) Cells from the third passage showed similar fibroblastic morphology. Bar: $100 \mu \mathrm{m}$.

no significant differences in the number and length of tubes between UCA-PSCs and UCV-PSCs $(P>0.05)$. These results demonstrated that UCA-PSCs and UCV-PSCs, especially UCA-PSCs, exhibited better angiogenic ability compared to the WJ-MSCs in vitro.

3.5. Higher Expression of CD146 and Jagged1 in UCA-PSCs. We carried out a genome-wide gene profile analysis to further investigate the biological characteristics of UCAPSCs, UCV-PSCs, and WJ-MSCs. As shown in the clustering analysis, based on 293 selected differentially expressed genes, UCA-PSCs were more closely related to UCV-PSCs than to WJ-MSCs (Figure 6(a)). In addition, many angiogenesisrelated genes, such as ISL1, JAG1, THBS1, CXCL12, CTGF, HIF1A, and ERAP1, were increased in UCA-PSCs and UCV-PSCs than in WJ-MSCs (Figure 6(b)). Furthermore,
Jagged1 expression was the highest in UCA-PSCs, followed by UCV-PSCs, and then WJ-MSCs (Figure 6(b)). Jagged 1 is well-known to play an important role in both physiological and pathological angiogenesis [23]. The Jagged1 mRNA levels were measured by qRT-PCR, and the results were in line with those of the microarray analysis (Figure 6(c)). The results of the western blot analysis also confirmed that UCA-PSCs had the highest CD146 and Jagged1 protein expression, followed by UCV-PSCs and WJ-MSCs. However, the protein level of Delta-like ligand (Dll4), another important ligand in Notch signals [24], revealed the opposite expression pattern among the three cell populations, which may be beneficial in angiogenesis (Figure 6(d)). Statistical analysis of the different protein levels of CD146 (Figure 6(e)), Jagged1 (Figure 6(f)), and Dll4 (Figure 6(g)) in UCA-PSCs, UCV-PSCs, and WJ-MSCs was also showed. 


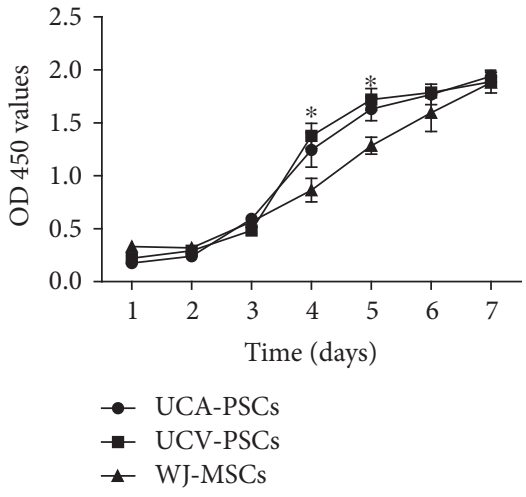

(a)

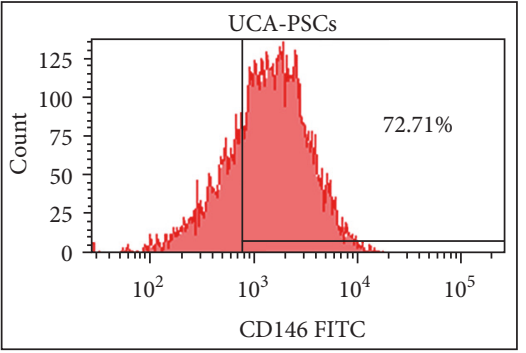

(c)

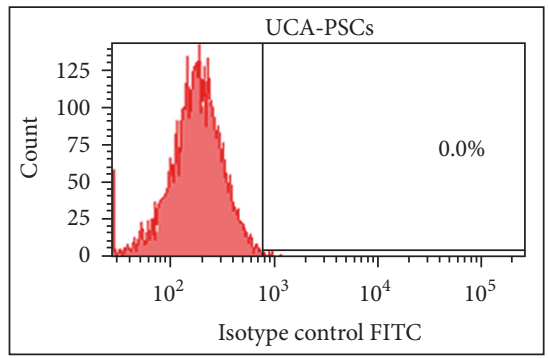

(f)

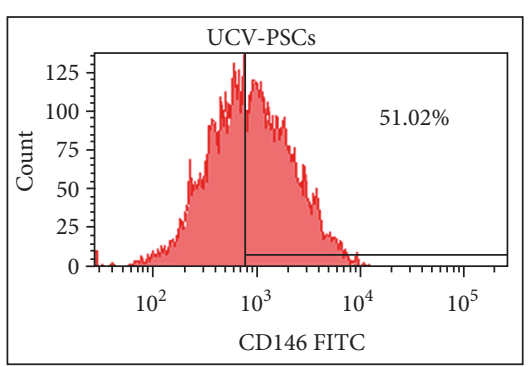

(d)

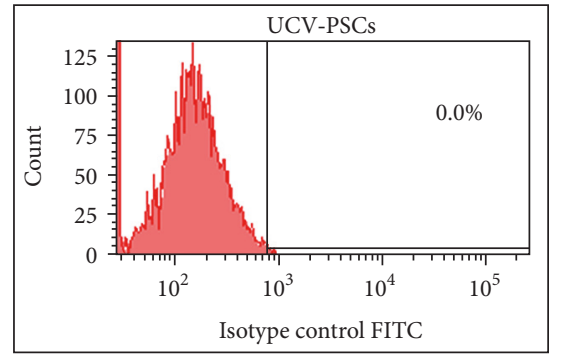

(g)

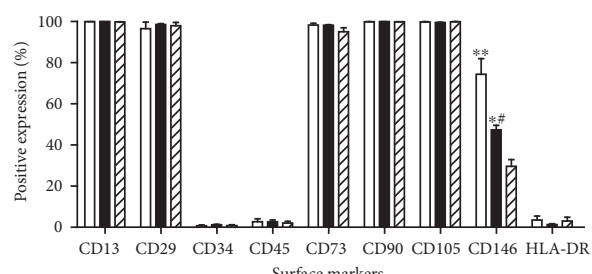

Surface markers

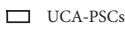

U] UT-MSCs (b)

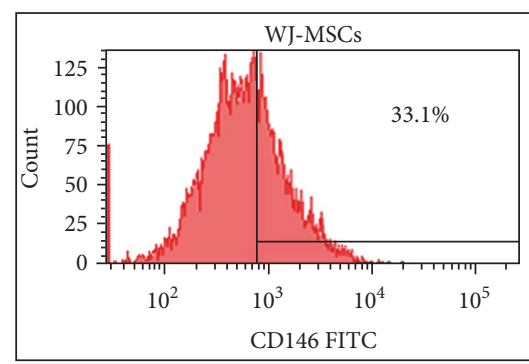

(e)

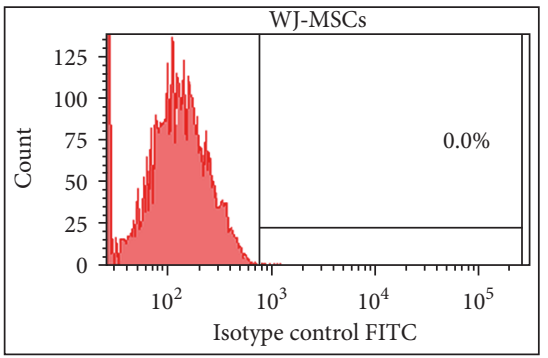

(h)

FIGURE 3: Proliferation and phenotype profile of umbilical cord artery perivascular stem cells (UCA-PSCs), umbilical cord vein perivascular stem cells (UCV-PSCs), and Wharton's jelly mesenchymal stem cells (WJ-MSCs). (a) Cell proliferation was continuously monitored for 7 days using cell-counting kit-8 (CCK-8), which showed that the number of UCA-PSCs was significantly higher than that of UCV-PSCs at days 4 and 5. However, the cell growth rate of UCA-PSCs, UCV-PSCs, and WJ-MSCs did not significantly differ. Bars represent the means \pm SE of three independent experiments performed in triplicate. ${ }^{*} P<0.05$, UCV-PSCs versus UCA-PSCs. (b) Profiles of cell surface epitopes in UCA-PSCs, UCV-PSCs, and WJ-MSCs. Abundance of cells positive for CD13, CD29, CD34, CD45, CD73, CD90, CD105, CD146, and HLA-DR, expressed as percentages, in UCA-PSCs, UCV-PSCs, and WJ-MSCs. Bars represent the means \pm SE of donor samples $(n=3) .{ }^{* *} P<0.01$, UCA-PSCs versus WJ-MSCs. ${ }^{*} P<0.05$, UCV-PSCs versus WJ-MSCs. ${ }^{*} P<0.05$, UCV-PSCs versus UCA-PSCs. (c-h) Representative flow cytometric plots, including isotype control (IgG-FITC) (f-h).

3.6. Knockdown of Jagged1 Decreased Tubule Formation in UCA-PSCs. To examine the role of Jagged 1 in angiogenesis, in particular the role of endogenous Jagged 1 in capillary tube formation of the three stem cells, siRNA was used to silence the expression of Jagged1 in UCA-PSCs, UCV-PSCs, and WJ-MSCs. The results showed that the depletion of Jagged 1 in UCA-PSCs, UCV-PSCs, and WJ-MSCs decreased Jagged1 mRNA level compared with the si-NC group (Figures $7(\mathrm{a})$, $7(\mathrm{~b})$, and $7(\mathrm{c}))$. Similarly, the three stem cells transfected with si-Jagged 1 expressed the lower protein level of Jagged1 at 72 hours after transfection (Figures 7(d), 7(e), and 7(f)). Transfection of si-Jagged 1 resulted in a $71.26 \%, 57.38 \%$, and $29.51 \%$ decrease of Jagged 1 expression in UCA-PSCs
(Figure $7(\mathrm{~g})$ ), UCV-PSCs (Figure $7(\mathrm{~h})$ ), and WJ-MSCs, respectively (Figure $7(\mathrm{i})$ ). Then, we determined the effect of Jagged1 knockdown on the angiogenic properties of the three stem cells in vitro. As shown in Figures $7(\mathrm{j}), 7(\mathrm{k})$, $7(\mathrm{l}), 7(\mathrm{~m}), 7(\mathrm{n})$, and 7(o), UCA-PSCs, UCV-PSCs, and WJ-MSCs transfected with si-Jagged1 or si-NC were cultured on Matrigel-coated plates for $3 \mathrm{~h}$. Representative images revealed that the number of tubule-like structures per random field was apparently lower in si-Jagged 1 group, compared with si-NC group in UCA-PSCs (Figures $7(\mathrm{j})$ and $7(\mathrm{~m})$ ), UCV-PSCs (Figures $7(\mathrm{k})$ and $7(\mathrm{n})$ ), and WJMSCs (Figures 7(l) and 7(o)). Statistical analysis showed that Jagged1 knockdown led to a significant reduction in 


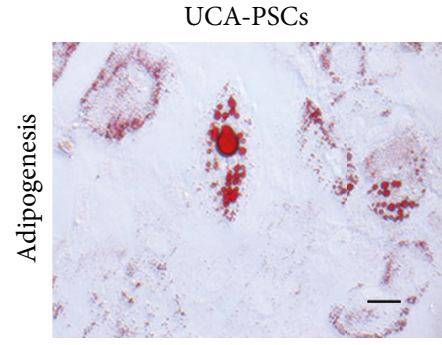

(a)

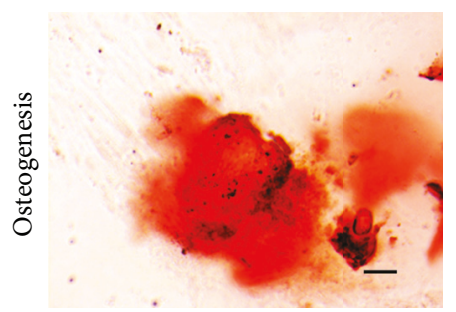

(d)

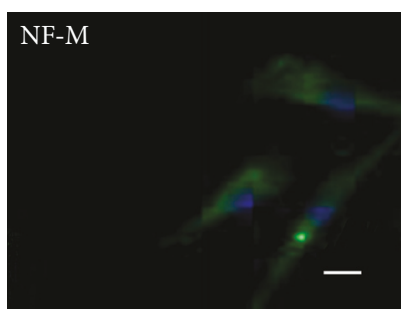

(g)

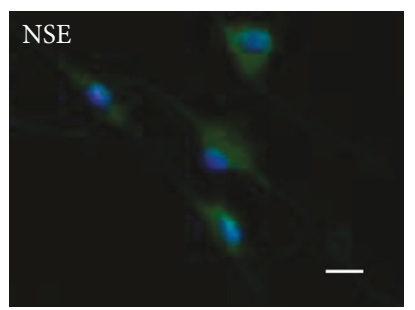

(j)

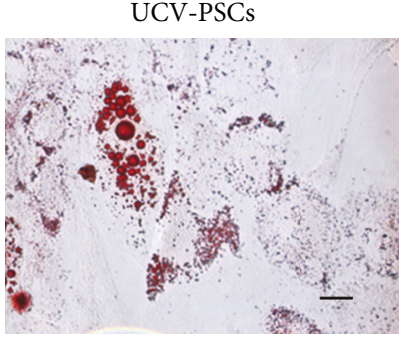

(b)

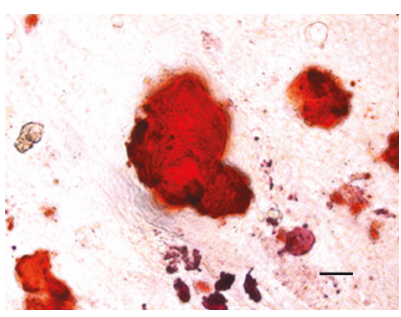

(e)

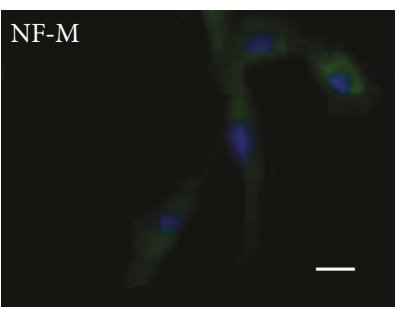

(h)

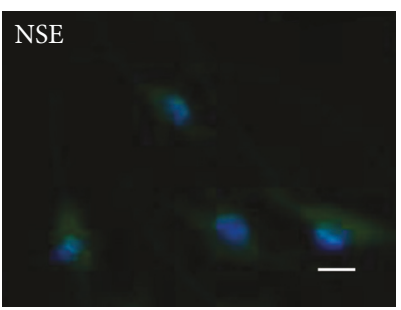

(k)

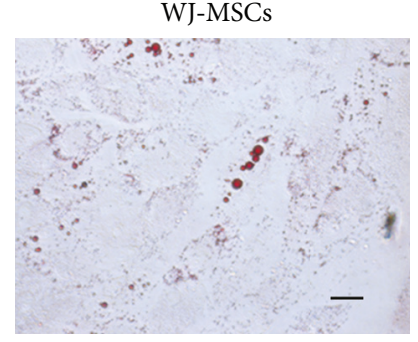

(c)

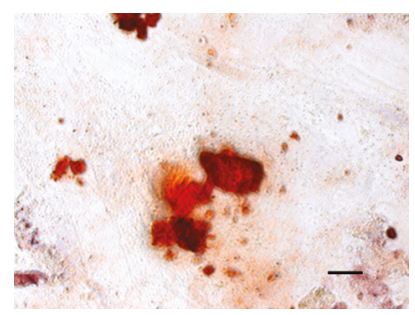

(f)

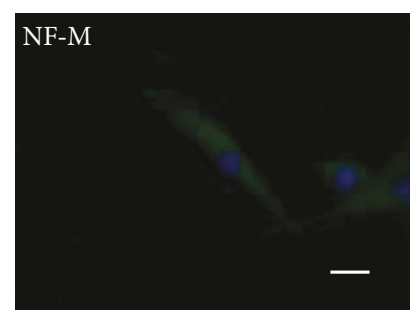

(i)

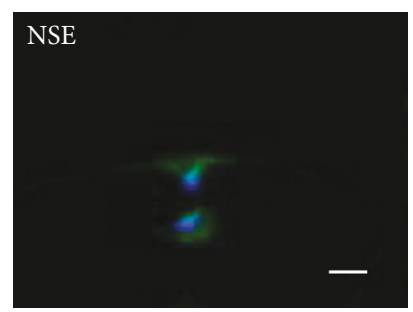

(1)

FIgURE 4: Differentiation of umbilical cord artery perivascular stem cells (UCA-PSCs), umbilical cord vein perivascular stem cells (UCV-PSCs), and Wharton's jelly mesenchymal stem cells (WJ-MSCs). (a-c) For adipogenic differentiation, the cells were cultured in adipogenic induction medium for 14 days. The formation of lipid droplets was confirmed by oil red $\mathrm{O}$ staining. (d-f) Cells were cultured in osteogenesis induction medium for 21 days. Calcium deposition was confirmed by alizarin red staining. (g-l) Differentiation of cells to neuronal lineage after $18 \mathrm{~h}$ preinduction and $36 \mathrm{~h}$ induction was confirmed by immunofluorescence staining of neurofilament medium polypeptide (g-i) and neuron-specific enolase $(\mathrm{j}-\mathrm{l})$. Bar: $10 \mu \mathrm{m}$.

the number of tubes per field in UCA-PSCs $(2.56 \pm 0.33$ versus $13.22 \pm 0.67 ; \quad P<0.001$, si-Jagged1 versus si-NC; Figure 7(p)), UCV-PSCs $(3.85 \pm 0.45$ versus $11.96 \pm 0.56$ $P<0.001$, si-Jagged1 versus si-NC; Figure $7(\mathrm{q}))$ and WJMSCs $(4.96 \pm 0.30$ versus $7.70 \pm 0.32 ; P<0.001$, si-Jagged 1 versus si-NC; Figure $7(\mathrm{r})$ ). In addition, compared to the siNC group, the total tube length of si-Jagged 1 group decreased by $72.74 \%$ in UCA-PSCs $(P<0.01$; Figure $7(\mathrm{~s})), 62.27 \%$ in UCV-PSCs $(P<0.01$; Figure $7(\mathrm{t}))$; and $23.38 \%$ in $\mathrm{WJ}-$ MSCs $(P>0.05$; Figure $7(\mathrm{u}))$. These data suggested that Jagged1 played a vital role in tube formation in the three stem cells.

\section{Discussion}

Human UC-derived mesenchymal stem cells are a promising versatile tool for regenerative medicine and immunotherapy [25]. This is the first study to compare the features of UCAPSCs, UCV-PSCs, and WJ-MSCs obtained from the same human UC. Our results revealed that UCA-PSCs expressed higher levels of CD146 than WJ-MSCs. Additionally, UCAPSCs and UCV-PSCs, especially UCA-PSCs, showed greater angiogenesis capacity and expressed higher levels of Jagged1, which is an important Notch ligand in angiogenesis. Our study demonstrated that the knockdown of Jagged1 


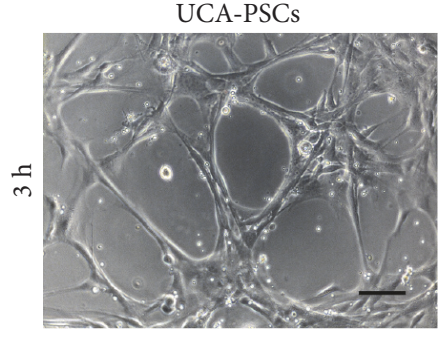

(a)

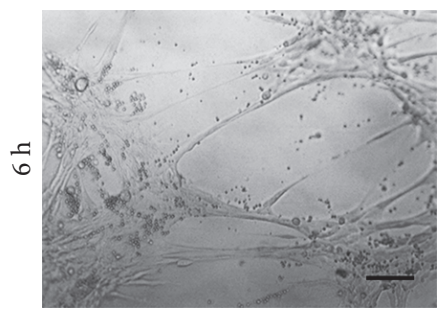

(d)

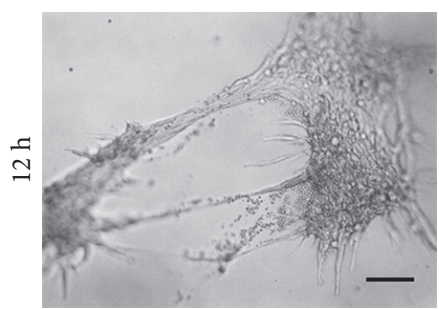

(g)

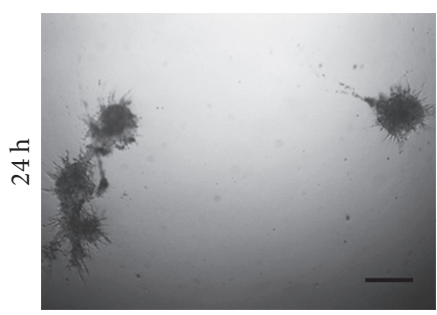

(j)

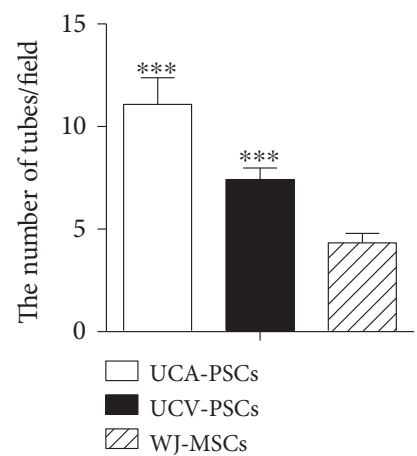

(m)

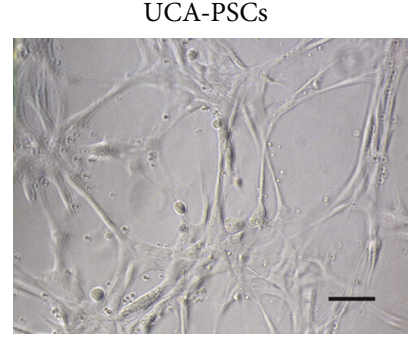

(b)

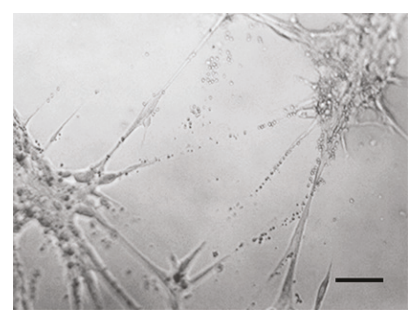

(e)

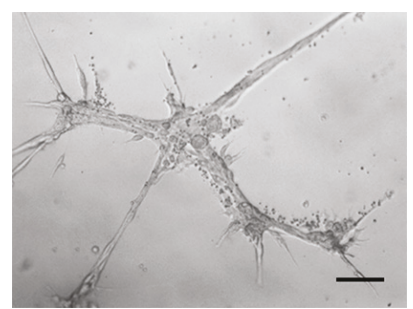

(h)

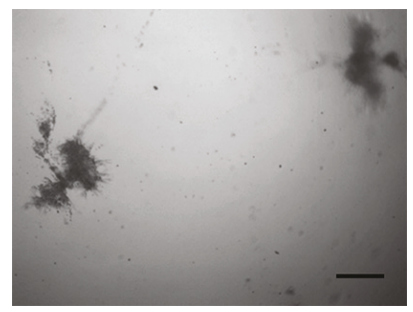

(k)

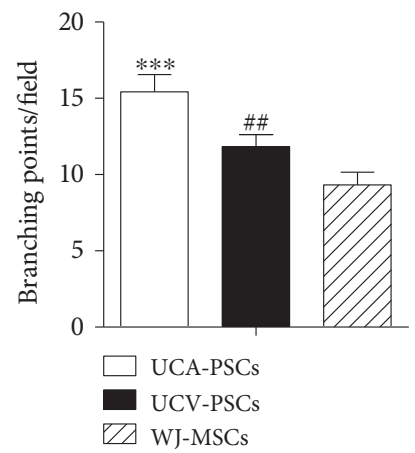

(n)

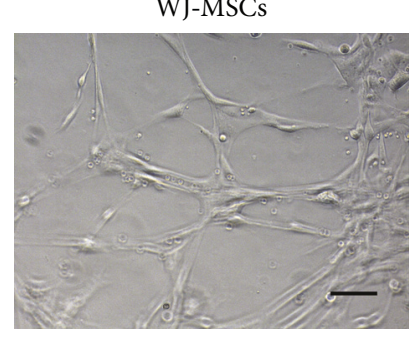

(c)

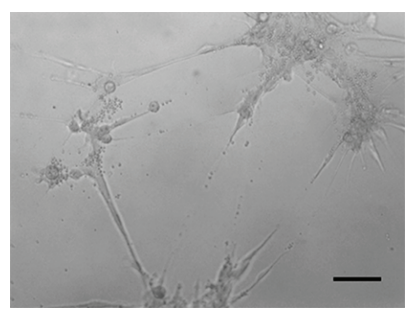

(f)

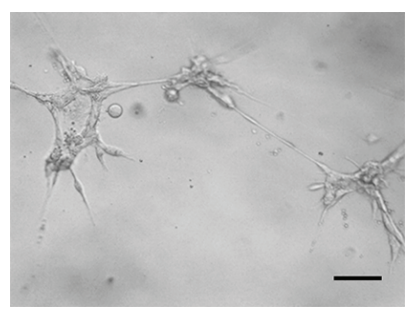

(i)

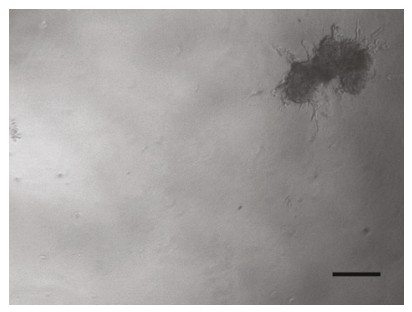

(1)

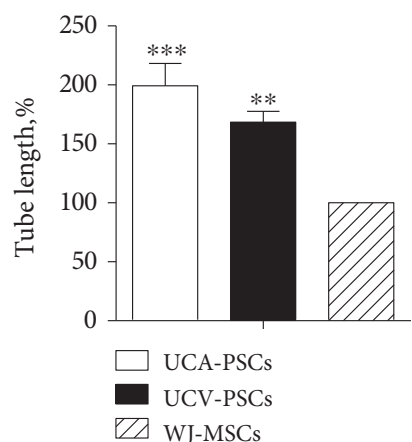

(o)

FIGURE 5: In vitro Matrigel tube formation assay. Umbilical cord artery perivascular stem cells (UCA-PSCs), umbilical cord vein perivascular stem cells (UCV-PSCs), and Wharton's jelly mesenchymal stem cells (WJ-MSCs). Cells $\left(1 \times 10^{4} /\right.$ well) were treated with a low concentration of glucose and plated on Matrigel in 96-well tissue culture plates. Tube formation was microscopically compared after $3 \mathrm{~h}(\mathrm{a}-\mathrm{c}), 6 \mathrm{~h}(\mathrm{~d}-\mathrm{f})$, $12 \mathrm{~h}(\mathrm{~g}-\mathrm{i})$, and $24 \mathrm{~h}(\mathrm{j}-\mathrm{l})$. Bar: $60 \mu \mathrm{m}$. The number of tubes $(\mathrm{m})$ and branching point per field (n) and the total length of tubes per field (o) were quantified $3 \mathrm{~h}$ after treatment by counting 3-5 random fields/well under the microscope (magnification: 100x). UCA-PSCs and UCV-PSCs, especially UCA-PSCs, displayed greater tube formation ability than WJ-MSCs. $n=5$. ${ }^{* * *} P<0.001$, versus WJ-MSCs. ${ }^{* *} P<0.01$, UCV-PSCs versus WJ-MSCs. ${ }^{\# \#} P<0.01$, UCV-PSCs versus UCA-PSCs. 


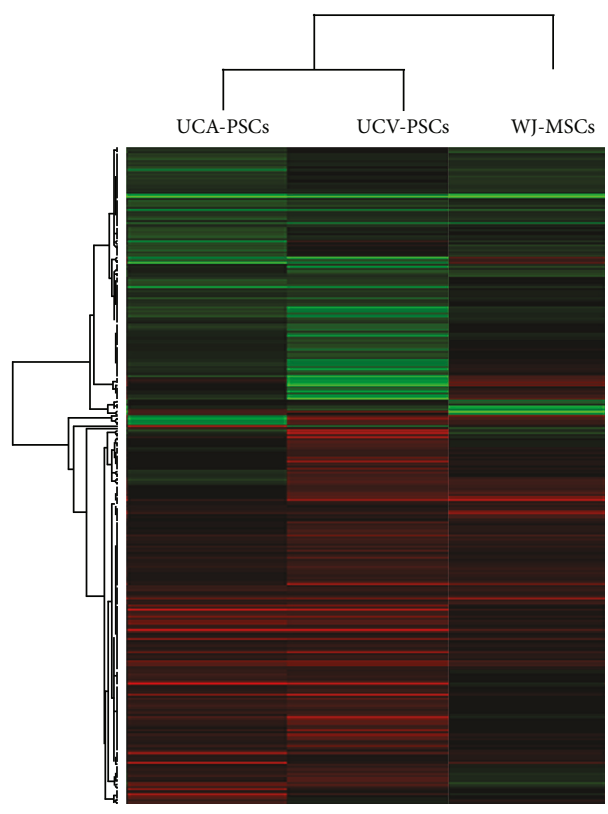

(a)

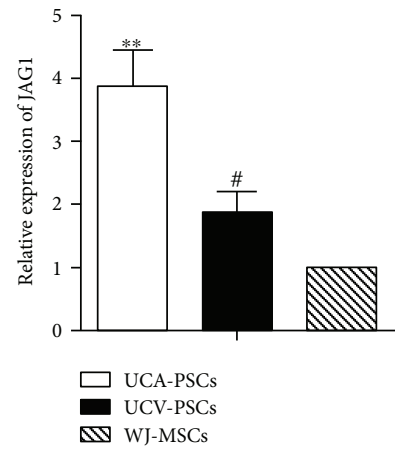

(c)

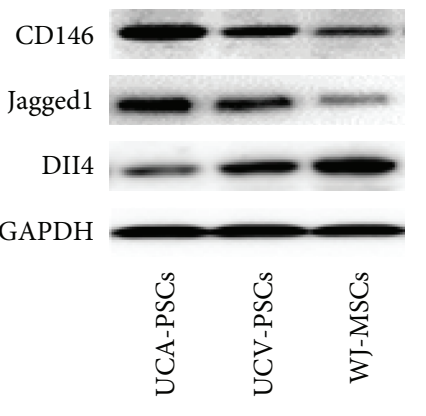

(d) (b)

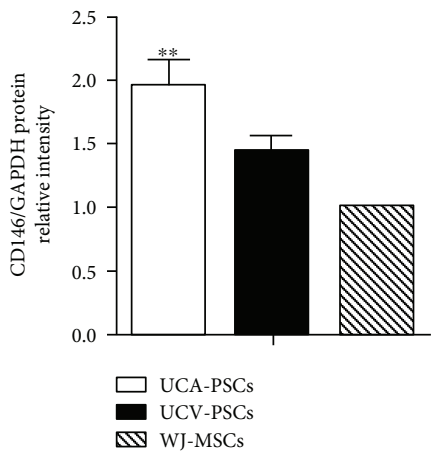

(e)

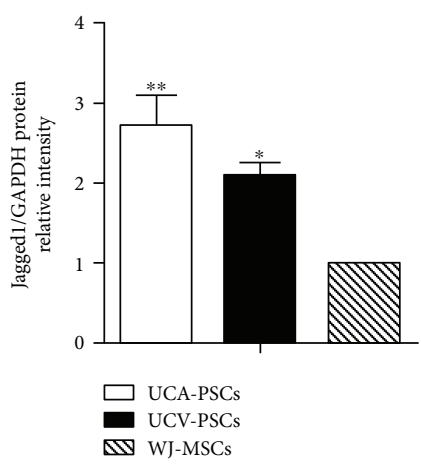

(f)

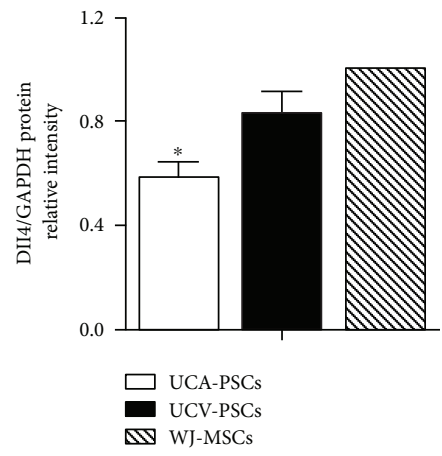

(g)

FIGURE 6: Expression of angiogenesis-related genes in the umbilical cord artery perivascular stem cells (UCA-PSCs), umbilical cord vein perivascular stem cells (UCV-PSCs), and Wharton's jelly mesenchymal stem cells (WJ-MSCs). (a) Comparison of gene expression patterns in UCA-PSCs, UCV-PSCs, and WJ-MSCs using microarray analysis. Red indicates upregulated genes while green indicates downregulated genes. (b) Gene expression heatmaps showed fold changes in the expression of a selection of genes involved in angiogenesis. (c) mRNA levels of Jagged1 in UCA-PSCs, UCV-PSCs, and WJ-MSCs measured by qRT-PCR. ${ }^{* *} P<0.01$, UCA-PSCs versus WJ-MSCs. ${ }^{\#} P<0.05$, UCV-PSCs versus UCA-PSCs. (d) Western blot analysis revealed that the CD146 and Jagged1 expression levels were the highest in UCA-PSCs, followed by UCV-PSCs and WJ-MSCs. While the Delta-like ligand (Dll4) protein displayed the opposite expression pattern in these three cell types. Quantitative analysis of CD146 (e), Jagged1 (f), and Dll4 (g) protein expression levels in UCA-PSCs, UCV-PSCs, and WJ-MSCs. Data are representative of three independent experiments performed in triplicate, and the expression of targeted protein was relative to the expression of GAPDH protein. ${ }^{* *} P<0.01$, versus WJ-MSCs. ${ }^{*} P<0.01$, UCV-PSCs versus UCA-PSCs. ${ }^{*} P<0.05$, versus WJ-MSCs. 


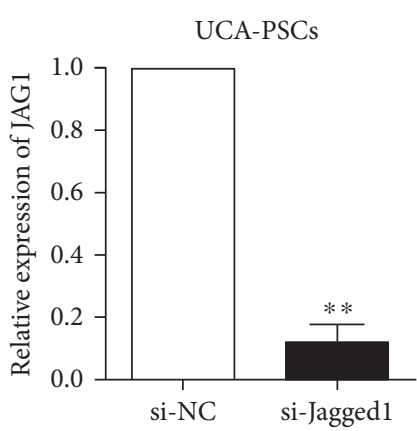

(a)

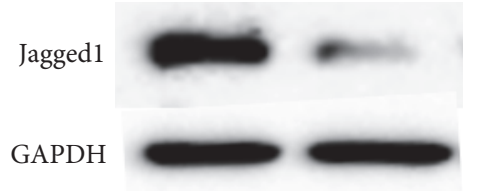

si-NC si-Jagged 1

(d)

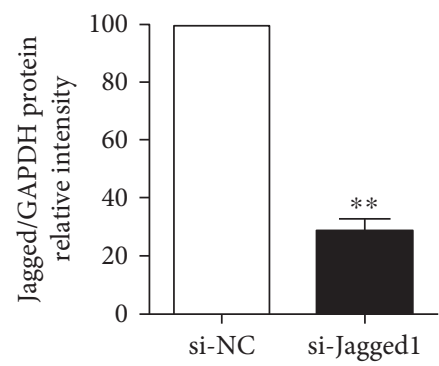

(g)

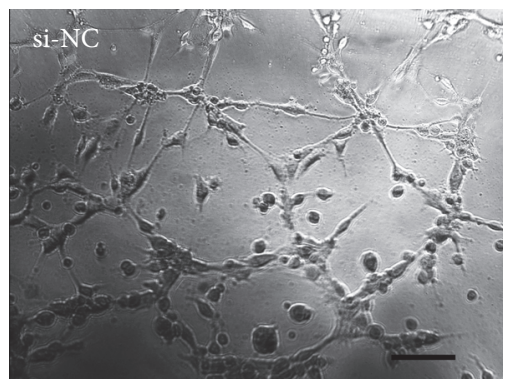

(j)

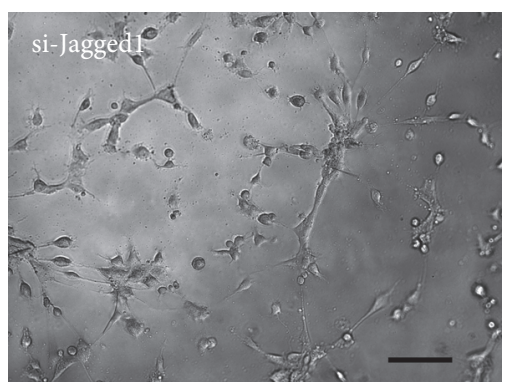

(m)

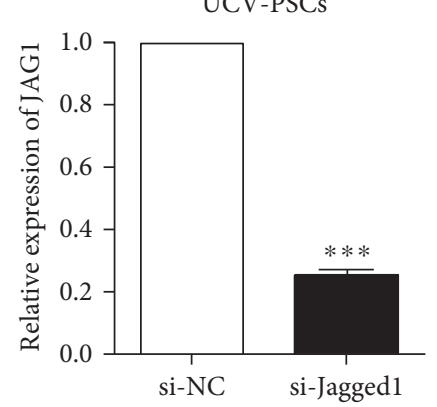

(b)

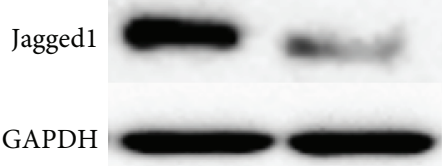

si-NC si-Jagged 1

(e)

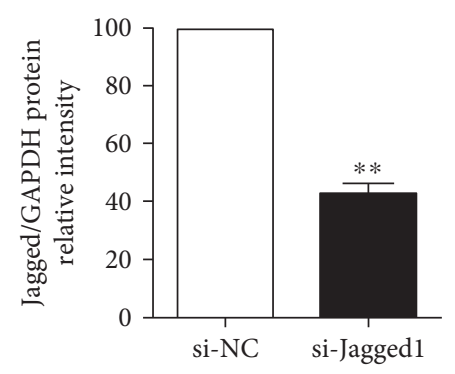

(h)

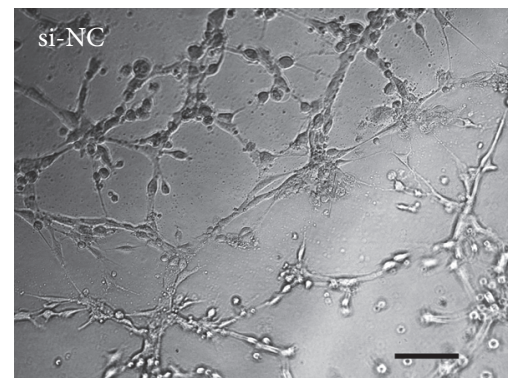

(k)

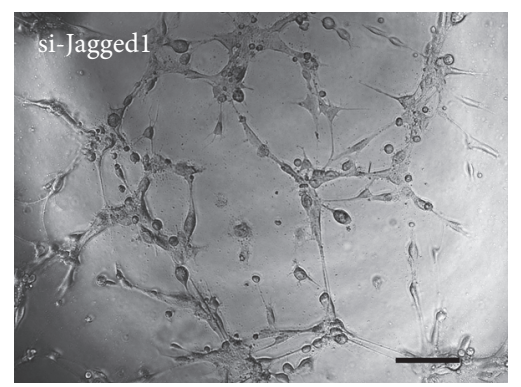

(n)

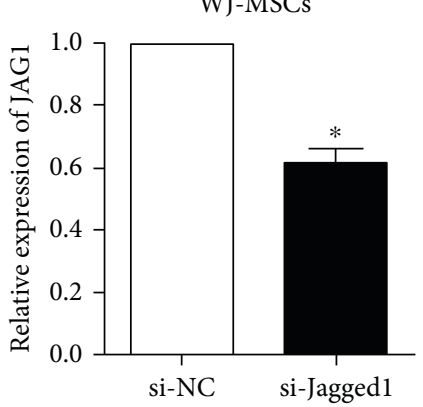

(c)

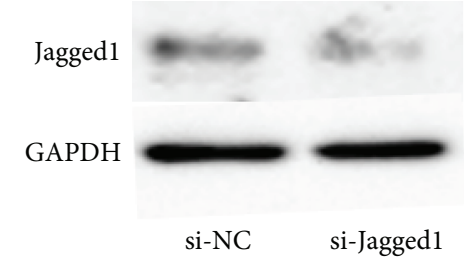

(f)

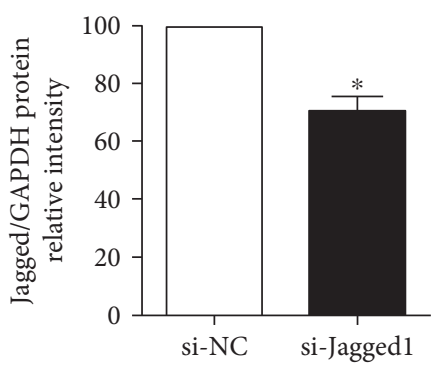

(i)

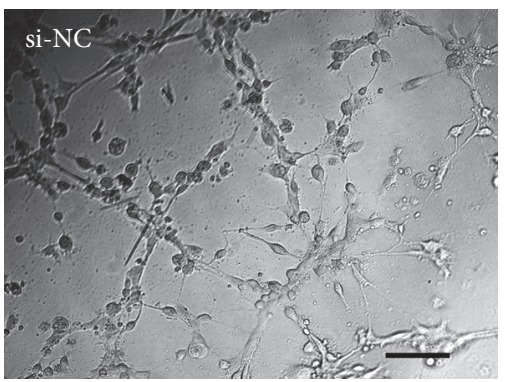

(1)

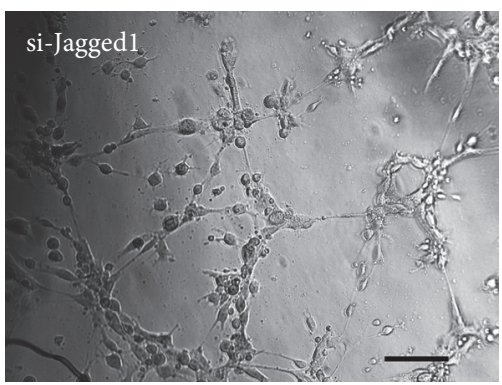

(o)

Figure 7: Continued. 


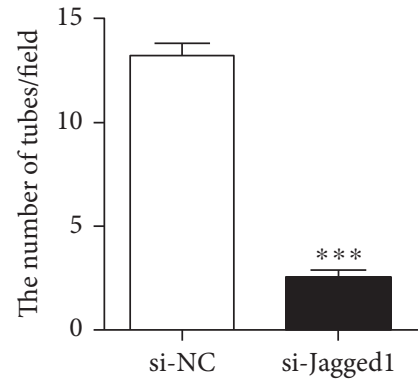

(p)

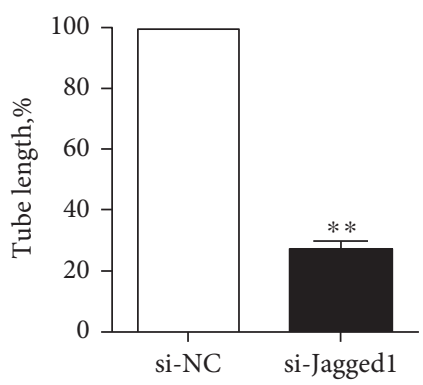

(s)

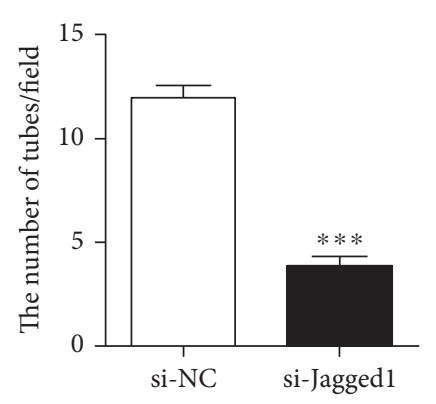

(q)

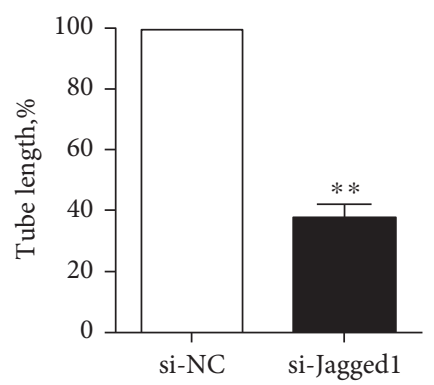

$(\mathrm{t})$

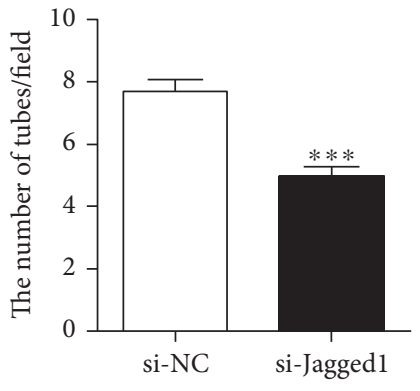

(r)

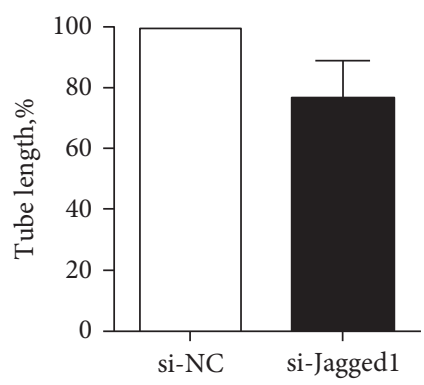

$(\mathrm{u})$

FIGURE 7: Knockdown of Jagged1 decreased tubule-like structures formation in the umbilical cord artery perivascular stem cells (UCA-PSCs), umbilical cord vein perivascular stem cells (UCV-PSCs), and Wharton's jelly mesenchymal stem cells (WJ-MSCs). (a-i) Jagged1 knockdown was effective. qRT-PCR analysis of Jagged1 mRNA levels in UCA-PSCs (a), UCV-PSCs (b), and WJ-MSCs (c) $48 \mathrm{~h}$ after the transfection with negative control siRNA (si-NC) or Jagged1 siRNA (si-Jagged1). ${ }^{* *} P<0.01$, si-Jagged1 versus si-NC; ${ }^{* * *} P<0.001$, si-Jagged1 versus si-NC; ${ }^{*} P<0.05$, si-Jagged 1 versus si-NC. Western blot analysis of Jagged 1 expression in UCA-PSCs (d), UCV-PSCs (e), and WJ-MSCs (f) $72 \mathrm{~h}$ after the transfection with si-NC or si-Jagged1. Quantitative analysis of Jagged1 protein expression levels in UCA-PSCs (g), UCV-PSCs (h), and WJ-MSCs (i) $72 \mathrm{~h}$ after the transfection with si-NC or si-Jagged1. Data are representative of three independent experiments performed in triplicate, and the expression of targeted protein was relative to the expression of GAPDH protein. ${ }^{* *} P<0.01$, si-Jagged 1 versus si-NC; ${ }^{*} P<0.05$, si-Jagged1 versus si-NC. Knockdown of Jagged1 decreased angiogenesis in UCA-PSCs, UCV-PSCs, and WJ-MSCs in vitro. ( $\mathrm{j}-\mathrm{O})$ Representative images of tubular structures. Bar: $50 \mu \mathrm{m}$. Tube formation assays were performed $72 \mathrm{~h}$ after transfection of si-NC or si-Jagged1. The number of tubes in UCA-PSCs (p), UCV-PSCs (q), and WJ-MSCs (r), together with the total length of tubes per field in UCA-PSCs (s), UCV-PSCs $(\mathrm{t})$, and WJ-MSCs $(\mathrm{u})$ were quantified $3 \mathrm{~h}$ after treatment by counting $3-5$ random fields/well under the microscope (magnification: 100x). $n=5 .{ }^{* * *} P<0.001$, si-Jagged1 versus si-NC; ${ }^{* *} P<0.01$, si-Jagged1 versus si-NC.

decreased tubule-like structure formation in UCA-PSCs, UCV-PSCs, and WJ-MSCs.

As a prerequisite to the identification of human perivascular cells, we used immunofluorescence assay to detect relevant marker combinations for this elusive cell population. It has been confirmed that all perivascular cells (pericytes) still display overextended culture, the markers their ancestors natively expressed in the tissue of origin (PDGF-R $\beta, N G 2$, $\alpha$-SMA, and CD146) [26]. In the present study, pericyte markers were detected in the perivascular region and the UCA had a higher proportion of cells expressing CD146 and NG2, indicating that there may be more perivascular stem cells surrounding UCA.

Meanwhile, the expression of CD146 was most notably elevated in UCA-PSCs and UCV-PSCs compared to WJMSCs, which was indicated not only by flow cytometry analysis of the harvested cell populations from the perivascular region but also by direct immunostaining of the human UC samples. CD146 is identified as a potential marker for multipotency [27]. The CD146 ${ }^{+}$subset of MSCs can longtime maintain the hematopoietic stem cells (HSCs) with engraftment and self-renewal ability [28]. In an experimental approach combining stringent cell purification by flow cytometry and differentiation in culture and in vivo, human $\mathrm{CD}_{146}{ }^{+}$perivascular cells represent the ubiquitous ancestors of MSCs [29]. Additionally, CD146 has been reported to play a crucial role in the vascular development [30]. A previous study found that knockdown of CD146 protein expression severely hindered vascular development, leading to poorly developed intersomitic vessels, with lack of blood flow through the intersomitic vessel region [31]. In addition, the gain-of-function analysis of CD146 in zebrafish found that enforcing expression of CD146 induced sprouting angiogenesis [32]. Moreover, as a novel VEGFR-2 coreceptor, CD146 is required in the promotion of endothelial cell migration and microvascular formation [33].

A complex labyrinth of blood vessels in the human body provides cells and tissues with the nutrients and oxygen needed for survival, proliferation, and a variety of physiological activities. The majority of the blood vessel network is considered to be built through angiogenic processes. Normal physiological angiogenesis is the formation of new blood vessels from preexisting vasculature, and it is a fundamental event during embryonic development, homeostasis, wound 
and fracture healing, and the growth and function of the female reproductive organs [34-36]. Thus, understanding the angiogenesis ability of these three MSC populations is vital in consideration of their clinical application. In the present study, we confirmed that UCA-PSCs had better tube formation capacity in vitro than WJ-MSCs. In addition to the increased number of tubes, branching points and total tube length per field, UCA-PSCs and UCV-PSCs, particularly UCA-PSCs, were also superior to WJ-MSCs in maintaining the stability of the tubes. Perivasculature has been considered to be the niche for various types of MSCs [37]. However, whether arteries, veins, and capillaries represent different MSC niches remains largely unknown. A recent study suggested that mouse incisor MSCs were localized around arterioles alone and not veins or capillaries and were regulated by the neurovascular bundle niche [38]. This finding may give a possible explanation as to why UCA-PSCs had a slight advantage over UCV-PSCs in terms of angiogenic capacity.

In addition to CD146, UCA-PSCs and UCV-PSCs both expressed higher levels of angiogenesis-related genes than WJ-MSCs, such as ISL1, JAG1, THBS1, CXCL12, CTGF, $H I F 1 A$, and ERAP1. It was reported that Jagged1 overexpression in tumor cells enhances neovascularization and tumor growth and that loss of Jagged1 in endothelial had an inhibitory effect on the neoangiogenic and maturation responses as well as an angiocrine effect in tumor cells [39]. Furthermore, mutations in the human Jagged1 gene cause Alagille syndrome, which involves complex cardiac defects and vascular anomalies [40]. Our data showed that knockdown of Jagged1 expression by siRNA in UCAPSCs, UCV-PSCs, and WJ-MSCs resulted in remarkably reduced tube formation in vitro. However, the knockdown efficiency in WJ-MSCs was lower compared to the other two kinds of cell, which may be explained by the low expression of endogenous Jagged1. During the process of angiogenesis, a well-regulated balance between the migration of tip cells and proliferation of stalk cells is essential for adequately shaped nascent sprouts [41]. Selecting the tip and the stalk fate is critical for developing a functional vessel and mediated by the Notch signaling pathway, a conserved cell-cell communication pathway activated through transinteractions between Notch ligands and receptors [42]. The Notch ligands Jagged1 and Dll4 have opposing effects on angiogenesis. Different signals might modulate angiogenesis by changing the ratio of Jagged1 and Dll4 expression, which integrated pro- or antiangiogenic signal into the selection of endothelial tip cells [43], which may be the cause of optimum angiogenic capacity of UCA-PSCs.

\section{Conclusions}

In summary, our results indicated for the first time that UCA-PSCs and UCV-PSCs, especially UCA-PSCs, had better angiogenesis capacity than WJ-MSCs in vitro. In addition, higher expression level of angiogenesis related genes, such as CD146 and Jagged1, was detected in UCA-PSCs.
These results offered a promising candidate, UCA-PSCs, for cell-based therapy for ischemia.

\section{Disclosure}

Lu Xu and Jianjun Zhou are co-first authors.

\section{Conflicts of Interest}

The authors declare that they have no competing interests.

\section{Acknowledgments}

This work was supported through Lijun Ding by grants from the Nature Science Foundation of China (81571391, 30900847) and Nanjing Medical Science Development Project (JQ2014004, ZKX16042). Haixiang Sun is supported by the Nature Science Foundation of China (31571189) and Strategic Priority Research Program of the Chinese Academy of Sciences (XDA01030501).

\section{References}

[1] A. Bronckaers, P. Hilkens, W. Martens et al., "Mesenchymal stem/stromal cells as a pharmacological and therapeutic approach to accelerate angiogenesis," Pharmacology \& Therapeutics, vol. 143, no. 2, pp. 181-196, 2014.

[2] M. L. Dominici, K. Le Blanc, I. Mueller et al., "Minimal criteria for defining multipotent mesenchymal stromal cells. The International Society for Cellular Therapy position statement," Cytotherapy, vol. 8, no. 4, pp. 315-317, 2006.

[3] M. F. Pittenger, A. M. Mackay, S. C. Beck et al., "Multilineage potential of adult human mesenchymal stem cells," Science, vol. 284, no. 5411, pp. 143-147, 1999.

[4] F. G. Teixeira, M. M. Carvalho, N. Sousa, and A. J. Salgado, "Mesenchymal stem cells secretome: a new paradigm for central nervous system regeneration," Cellular and Molecular Life Sciences, vol. 70, no. 20, pp. 3871-3882, 2013.

[5] M. M. Carvalho, F. G. Teixeira, R. L. Reis, N. Sousa, and A. J. Salgado, "Mesenchymal stem cells in the umbilical cord: phenotypic characterization, secretome and applications in central nervous system regenerative medicine," Current Stem Cell Research \& Therapy, vol. 6, no. 3, pp. 221-228, 2011.

[6] D. W. Kim, M. Staples, K. Shinozuka, P. Pantcheva, S. D. Kang, and C. V. Borlongan, "Wharton's jelly-derived mesenchymal stem cells: phenotypic characterization and optimizing their therapeutic potential for clinical applications," International Journal of Molecular Sciences, vol. 14, no. 6, pp. 1169211712, 2013.

[7] M. T. Conconi, R. Di Liddo, M. Tommasini, C. Calore, and P. P. Parnigotto, "Phenotype and differentiation potential of stromal populations obtained from various zones of human umbilical cord: an overview," The Open Tissue Engineering and Regenerative Medicine Journal, vol. 4, no. 1, pp. 6-20, 2011.

[8] A. K. Batsali, M. C. Kastrinaki, H. A. Papadaki, and C. Pontikoglou, "Mesenchymal stem cells derived from Wharton's jelly of the umbilical cord: biological properties and emerging clinical applications," Current Stem Cell Research \& Therapy, vol. 8, no. 2, pp. 144-155, 2013. 
[9] M. M. Carvalho, F. G. Teixeira, R. L. Reis, N. Sousa, and A. J. Salgado, "Mesenchymal stem cells in the umbilical cord: phenotypic characterization, secretome and applications in central nervous system regenerative medicine," Current Stem Cell Research \& Therapy, vol. 6, no. 3, pp. 221-228, 2011.

[10] D. Baksh, R. Yao, and R. S. Tuan, "Comparison of proliferative and multilineage differentiation potential of human mesenchymal stem cells derived from umbilical cord and bone marrow," Stem Cells, vol. 25, no. 6, pp. 1384-1392, 2007.

[11] R. Shohara, A. Yamamoto, S. Takikawa et al., "Mesenchymal stromal cells of human umbilical cord Wharton's jelly accelerate wound healing by paracrine mechanisms," Cytotherapy, vol. 14, no. 10, pp. 1171-1181, 2012.

[12] M. Crisan, M. Corselli, W. C. Chen, and B. Péault, "Perivascular cells for regenerative medicine," Journal of Cellular and Molecular Medicine, vol. 16, no. 12, pp. 2851-2860, 2012.

[13] M. Corselli, C. W. Chen, M. Crisan, L. Lazzari, and B. Péault, "Perivascular ancestors of adult multipotent stem cells," Arterioscler Thrombosis and Vascular Biology, vol. 30, no. 6, pp. 1104-1109, 2010.

[14] B. Sacchetti, A. Funari, S. Michienzi et al., "Self-renewing osteoprogenitors in bone marrow sinusoids can organize a hematopoietic microenvironment," Cell, vol. 131, no. 2, pp. 324-336, 2007.

[15] G. T. Huang, T. Yamaza, L. D. Shea et al., "Stem/progenitor cell-mediated de novo regeneration of dental pulp with newly deposited continuous layer of dentin in an in vivo model," Tissue Engineering. Part A, vol. 16, no. 2, pp. 605-615, 2010.

[16] W. Tang, D. Zeve, J. M. Suh et al., "White fat progenitor cells reside in the adipose vasculature," Science, vol. 322, no. 5901, pp. 583-586, 2008.

[17] A. Dar, H. Domev, O. Ben-Yosef et al., "Multipotent vasculogenic pericytes from human pluripotent stem cells promote recovery of murine ischemic limb," Circulation, vol. 125, no. 1, pp. 87-99, 2012.

[18] P. Campagnolo, D. Cesselli, A. A. Zen et al., "Human adult vena saphena contains perivascular progenitor cells endowed with clonogenic and proangiogenic potential," Circulation, vol. 121, no. 15, pp. 1735-1745, 2010.

[19] H. Chen, N. Zhang, T. Li et al., "Human umbilical cord Wharton's jelly stem cells: immune property genes assay and effect of transplantation on the immune cells of heart failure patients," Cellular Immunology, vol. 276, no. 1-2, pp. 83-90, 2012.

[20] C. Mennan, K. Wright, A. Bhattacharjee, B. Balain, J. Richardson, and S. Roberts, "Isolation and characterisation of mesenchymal stem cells from different regions of the human umbilical cord," BioMed Research International, vol. 2013, Article ID 916136, 8 pages, 2013.

[21] T. Nagamura-Inoue and H. He, "Umbilical cord-derived mesenchymal stem cells: their advantages and potential clinical utility," World Journal of Stem Cells, vol. 6, no. 2, pp. 195-202, 2014.

[22] W. P. Tsang, Y. Shu, P. L. Kwok et al., "CD146 ${ }^{+}$human umbilical cord perivascular cells maintain stemness under hypoxia and as a cell source for skeletal regeneration," PloS One, vol. 8, no. 10, article e76153, 2013.

[23] M. Boareto, M. K. Jolly, E. Ben-Jacob, and J. N. Onuchic, "Jagged mediates differences in normal and tumor angiogenesis by affecting tip-stalk fate decision," Proceedings of the
National Academy of Sciences United States of America, vol. 112, no. 29, pp. E3836-E3844, 2015.

[24] M. Nemir, M. Metrich, I. Plaisance et al., "The Notch pathway controls fibrotic and regenerative repair in the adult heart," European Heart Journal, vol. 35, no. 32, pp. 2174-2185, 2014.

[25] N. Watson, R. Divers, R. Kedar et al., "Discarded Wharton jelly of the human umbilical cord: a viable source for mesenchymal stromal cells," Cytotherapy, vol. 17, no. 1, pp. 18-24, 2015.

[26] M. Crisan, S. Yap, L. Casteilla et al., "A perivascular origin for mesenchymal stem cells in multiple human organs," Cell Stem Cell, vol. 3, no. 3, pp. 301-313, 2008.

[27] K. C. Russell, D. G. Phinney, M. R. Lacey, B. L. Barrilleaux, K. E. Meyertholen, and K. C. O'Connor, "In vitro highcapacity assay to quantify the clonal heterogeneity in trilineage potential of mesenchymal stem cells reveals a complex hierarchy of lineage commitment," Stem Cells, vol. 28, no. 4, pp. 788-798, 2010.

[28] M. Corselli, M. Crisan, I. R. Murray et al., "Identification of perivascular mesenchymal stromal/stem cells by flow cytometry," Cytometry. Part A, vol. 83, no. 8, pp. 714-720, 2013.

[29] M. Corselli, C. J. Chin, C. Parekh et al., "Perivascular support of human hematopoietic stem/progenitor cells," Blood, vol. 121, no. 15, pp. 2891-2901, 2013.

[30] Z. Wang and X. Yan, "CD146, a multi-functional molecule beyond adhesion," Cancer Letters, vol. 330, no. 2, pp. 150162, 2013.

[31] B. Chan, S. Sinha, D. Cho, R. Ramchandran, and V. P. Sukhatme, "Critical roles of CD146 in zebrafish vascular development," Developmental Dynamics, vol. 232, no. 1, pp. 232-244, 2005.

[32] J. H. So, S. K. Hong, H. T. Kim et al., "Gicerin/Cd146 is involved in zebrafish cardiovascular development and tumor angiogenesis," Genes to Cells, vol. 15, no. 11, pp. 1099-1110, 2010.

[33] T. Jiang, J. Zhuang, H. Duan et al., "CD146 is a coreceptor for VEGFR-2 in tumor angiogenesis," Blood, vol. 120, no. 11, pp. 2330-2339, 2012.

[34] S. V. Bhadada, B. R. Goyal, and M. M. Patel, "Angiogenic targets for potential disorders," Fundamental and Clinic Pharmacology, vol. 25, no. 1, pp. 29-47, 2011.

[35] P. Carmeliet and R. K. Jain, "Principles and mechanisms of vessel normalization for cancer and other angiogenic diseases," Nature Reviews. Drug Discovery, vol. 10, no. 6, pp. 417-427, 2011.

[36] R. Benedito and M. Hellström, "Notch as a hub for signaling in angiogenesis," Experimental Cell Research, vol. 319, no. 9, pp. 1281-1288, 2013.

[37] N. J. Krautler, V. Kana, J. Kranich et al., "Follicular dendritic cells emerge from ubiquitous perivascular precursors," Cell, vol. 150, no. 1, pp. 194-206, 2012.

[38] H. Zhao, J. Feng, K. Seidel et al., "Secretion of shh by a neurovascular bundle niche supports mesenchymal stem cell homeostasis in the adult mouse incisor," Cell Stem Cell, vol. 14, no. 2, pp. 160-173, 2014.

[39] A. R. Pedrosa, A. Trindade, C. Carvalho et al., "Endothelial Jagged1 promotes solid tumor growth through both proangiogenic and angiocrine functions," Oncotarget, vol. 6, no. 27, pp. 24404-24423, 2015.

[40] L. Li, J. M. Miano, P. Cserjesi, and E. N. Olson, "SM22 alpha, a marker of adult smooth muscle, is expressed in 
multiple myogenic lineages during embryogenesis," Circulation Research, vol. 78, no. 2, pp. 188-195, 1996.

[41] I. Geudens and H. Gerhardt, "Coordinating cell behaviour during blood vessel formation," Development, vol. 138, no. 21, pp. 4569-4583, 2011.

[42] R. Kopan and M. X. Ilagan, "The canonical Notch signaling pathway: unfolding the activation mechanism," Cell, vol. 137, no. 2, pp. 216-233, 2009.

[43] R. Benedito, C. Roca, I. Sörensen et al., “The notch ligands Dll4 and Jagged1 have opposing effects on angiogenesis," Cell, vol. 137, no. 6, pp. 1124-1135, 2009. 

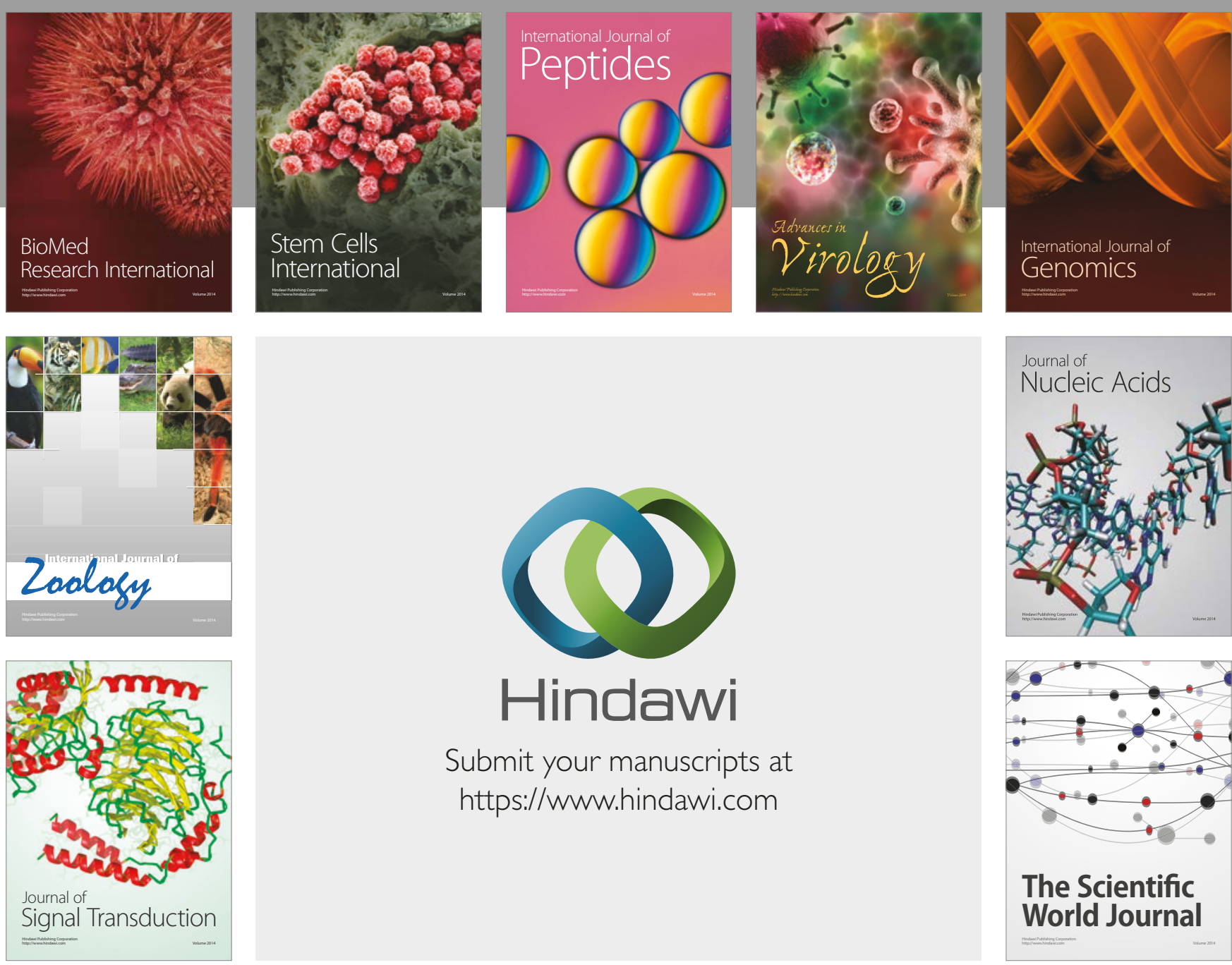

Submit your manuscripts at

https://www.hindawi.com
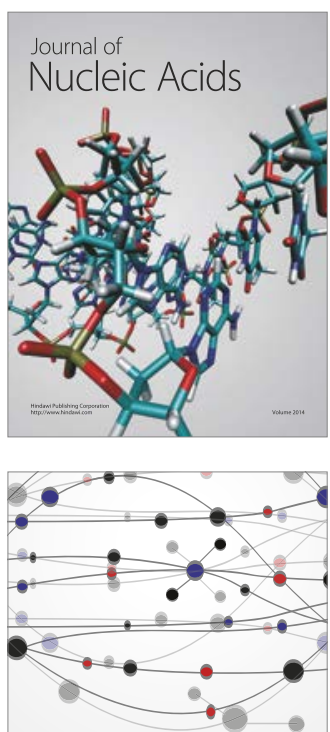

The Scientific World Journal

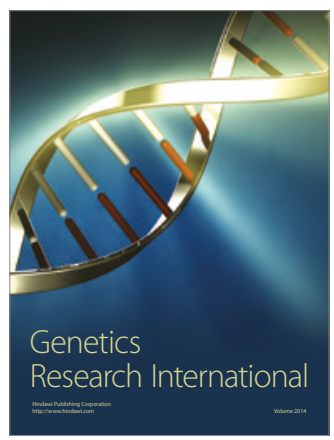

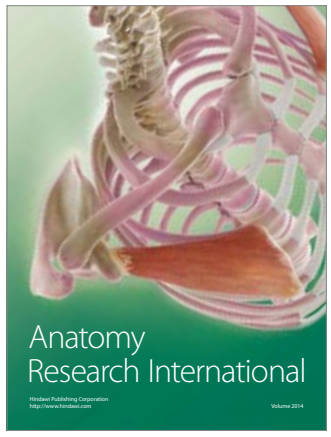

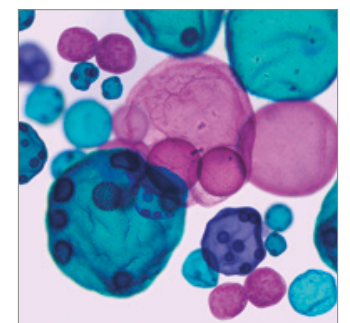

International Journal of Microbiology
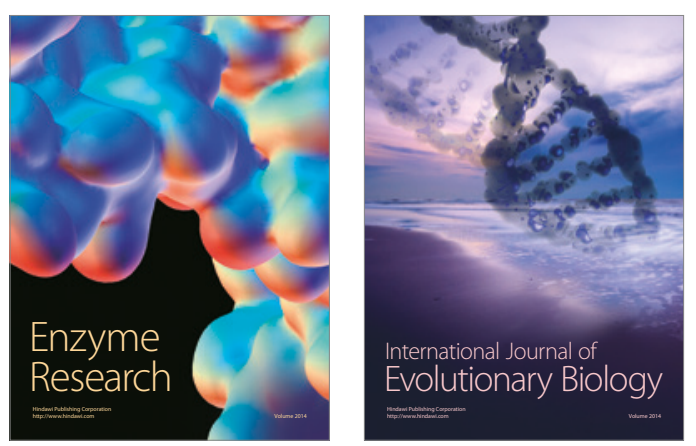
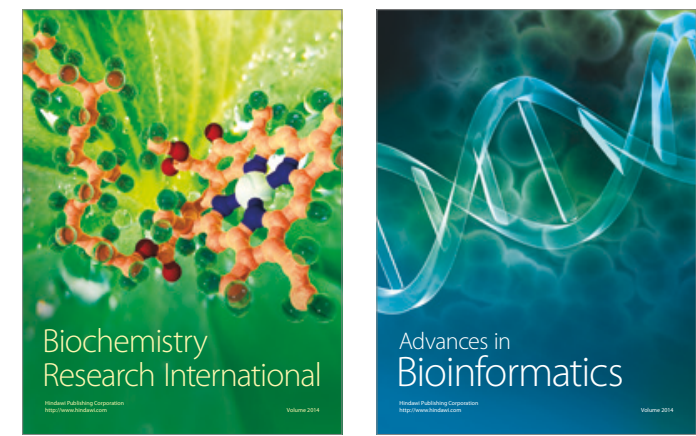

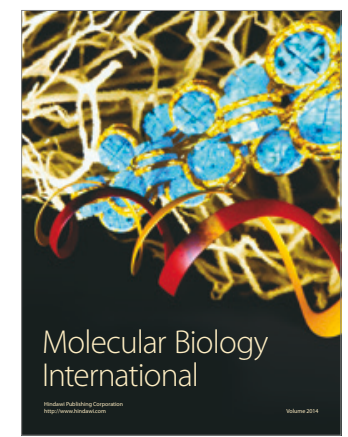

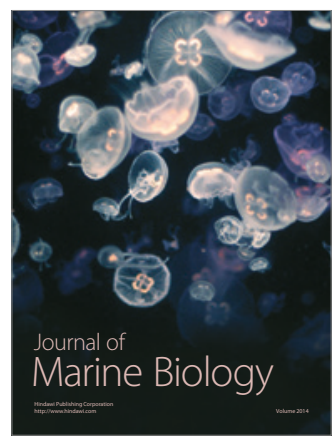

